NBER WORKING PAPER SERIES

FINANCIAL CENTRALITY AND LIQUIDITY PROVISION

Arun G. Chandrasekhar

Robert Townsend

Juan Pablo Xandri

Working Paper 24406

http://www.nber.org/papers/w24406

\author{
NATIONAL BUREAU OF ECONOMIC RESEARCH \\ 1050 Massachusetts Avenue \\ Cambridge, MA 02138 \\ March 2018
}

We thank Ben Golub, Matthew Jackson, and Juan Passadore for helpful discussions as well as seminar participants at NYU, Princeton, SAET Faro, Minnesota, MIT, and various other seminars and conferences. Chandrasekhar is grateful for support from the National Science Foundation GFRP and Townsend is grateful for support from the NICHD. The views expressed herein are those of the authors and do not necessarily reflect the views of the National Bureau of Economic Research.

NBER working papers are circulated for discussion and comment purposes. They have not been peer-reviewed or been subject to the review by the NBER Board of Directors that accompanies official NBER publications.

(C) 2018 by Arun G. Chandrasekhar, Robert Townsend, and Juan Pablo Xandri. All rights reserved. Short sections of text, not to exceed two paragraphs, may be quoted without explicit permission provided that full credit, including ()$^{\text {notice, }}$ is given to the source. 
Financial Centrality and Liquidity Provision

Arun G. Chandrasekhar, Robert Townsend, and Juan Pablo Xandri

NBER Working Paper No. 24406

March 2018

JEL No. D14,E44,G01,L14,O16

\begin{abstract}
We study an endowment economy in which agents face income risk, as if uncertain returns on a portfolio, and agents can only make transfers in states when they are actively participating in the market. Besides income risk, agents also have limited and stochastic market access, with a probability distribution governed by an underlying social network. While network connections may serve to dissipate shocks, they may also provide obstacles to the sharing of risk, as when participation frictions are generated through the network.
\end{abstract}

We identify and quantify the value of key players in terms of whether they are likely to be able to smooth the resulting market participation risk and how valuable that smoothing would be when they are there. We define financial centrality in economic terms, given the model, as the ex ante marginal social value of injecting an infinitesimal amount of liquidity to the agent.

We show that the most financially central agents are not only those who trade often - as in standard network models - but are more likely to trade when there are few traders, when income risk is high, when income shocks are positively correlated, when attitudes toward risk are more sensitive in the aggregate, when there are distressed institutions, and when there are tail risks. We extend our framework to allow for endogenous market participation. Observational evidence from village risk sharing network data is consistent with our model.

Arun G. Chandrasekhar

Department of Economics

Stanford University

579 Serra Mall

Stanford, CA 94305

and NBER

arungc@stanford.edu

Robert Townsend

Department of Economics, E52-538

MIT

77 Massachusetts Avenue

Cambridge, MA 02139

and NBER

rtownsen@mit.edu
Juan Pablo Xandri

Department of Economics

Princeton University

Fisher Hall, Office 212

Princeton, NJ 08544

jxandri@princeton.edu 


\title{
FINANCIAL CENTRALITY AND LIQUIDITY PROVISION
}

\author{
ARUN G. CHANDRASEKHAR ${ }^{\ddagger}$, ROBERT M. TOWNSEND ${ }^{\S}$, AND JUAN PABLO XANDRI ${ }^{\star}$
}

ABSTRACT. We study an endowment economy in which agents face income risk, as if uncertain returns on a portfolio, and agents can only make transfers in states when they are actively participating in the market. Besides income risk, agents also have limited and stochastic market access, with a probability distribution governed by an underlying social network. While network connections may serve to dissipate shocks, they may also provide obstacles to the sharing of risk, as when participation frictions are generated through the network.

We identify and quantify the value of key players in terms of whether they are likely to be able to smooth the resulting market participation risk and how valuable that smoothing would be when they are there. We define financial centrality in economic terms, given the model, as the ex ante marginal social value of injecting an infinitesimal amount of liquidity to the agent.

We show that the most financially central agents are not only those who trade often -- as in standard network models -- but are more likely to trade when there are few traders, when income risk is high, when income shocks are positively correlated, when attitudes toward risk are more sensitive in the aggregate, when there are distressed institutions, and when there are tail risks. We extend our framework to allow for endogenous market participation. Observational evidence from village risk sharing network data is consistent with our model.

JEL Classification Codes: D14, E44, G01, L14, O16

KEYWORDS: Financial networks, networks, market participation, liquidity injection

\section{INTRODUCTION}

We focus on a measure of financial centrality and the identification of key players in financial markets in environments in which disruptions to markets take the form of shocks to market participation.

These shocks are consistent with the foundations of several types of models that are widely used to think about financial markets. The first class of models include search frictions with bilateral and stochastic matching as in Duffie et al. (2005), and, in particular, directed search models in which subsets of agents group together facing a tradeoff between offers and matching

Date: March 15, 2018.

We thank Ben Golub, Matthew Jackson, and Juan Passadore for helpful discussions as well as seminar participants at NYU, Princeton, SAET Faro, Minnesota, MIT, and various other seminars and conferences. Chandrasekhar is grateful for support from the National Science Foundation GFRP and Townsend is grateful for support from the NICHD.

${ }^{\ddagger}$ Stanford University. Email: arungc@stanford.edu .

${ }^{\S}$ Massachusetts Institute of Technology. Email: rtownsen@mit.edu .

${ }^{\star}$ Princeton University. Email: jxandri@princeton.edu. 
rates as in Armenter and Lester (2015). The second class of models include market participation models used in finance and monetary economics, in particular, when the number and composition of traders who can deal with one another is stochastic as in Kiyotaki and Wright (1989), Trejos and Wright (1995), Freeman (1996) and Green and Zhou (2002), Santos and Woodford (1997). We focus especially on the resulting risk of thin markets, as this is a particular concern to the traders individually and to overall social welfare. Putting this another way, we focus not only on liquidity shocks but also insurance against them. Broker dealers are such key traders in the private sector, as in Cocco et al. (2009) where relationships among dealers with less correlated shocks allows insurance against shortage of funds in the reserve maintenance period.

Much of the financial network literature posits unanticipated shocks which can hit the balance sheet of selected traders ex-post and focuses how these adverse shocks may spread through the financial system via the network. Empirical studies on such networks include Upper and Worms (2004)Bech and Atalay (2010), Boss et al. (2004), Cohen-Cole et al. (2013), Craig and Von Peter (2014), Cont et al. (2013), Langfield et al. (2014), Jaramillo (2012), and others. The economic and/or network theory is developed in Allen and Gale (2000), Freixas et al. (2000) and Eisenberg and Noe (2001), and more recently in Gai and Kapadia (2010), Battiston et al. (2012), Acemoglu et al. (2015), and Elliott et al. (2014). See Allen and Babus (2009) and Summer (2013) for surveys.

Many of the papers in this literature, motivated by the financial crisis, are concerned with how the pattern of connections and obligations across banks could induce contagion. In this sense interconnectedness is viewed as problematic and indeed financial systems are being regulated accordingly, identifying institutions regarded as systematically important and implementing requirements that have the intent of limiting their size and/or limiting the exposure of the market to them, as in risk capital and liquidity ratios. But as Summer (2013) notes, many of the models of this literature miss the intuition provided by the standard risk sharing model. A shock to a highly interconnected system might be more easily dissipated because it can be absorbed by more parties (Acemoglu et al., 2010; Elliott et al., 2014). This idea was key in the original work of Allen and Gale (2000); when they allow a complete contract structure, they do not get contagion, as they note. Thus the actual network of exposures may be conducive to the sharing of idiosyncratic risk.

On the other hand, depending on how it is modeled, the network structure may provide an obstacle to risk-sharing and leave agents exposed (Ambrus et al., 2010). Indeed in key examples, we link the network structure to the way stochastic market participation shocks are generated. We can then distinguish across institutions or traders by their position in a network, and we show that financial centrality matters. However, our notion of ex-ante financial centrality, associated with a constrained Pareto outcome in the face of these market shocks, is different from the typical graph-theoretic definitions of network centrality and those implemented in the empirical literature. For us degree centrality, eigenvalue centrality, betweenness, closeness, and other measures of network centrality are potentially different from the correct measure of 
financial centrality in our risk sharing environment. For example, degree centrality matters less if being in such a central position and likely to be in the market means that it brings many others to the market at the same time. Likewise, if market participation is limited, key players are those that bring liquidity to the market, that is, are those who are likely to be around precisely when the the number of traders is small.

More specifically, as trade is inherently limited by participation frictions, idiosyncratic shocks do not typically net to zero. Thus there can be considerable market risk: idiosyncratic shocks are a source of aggregate risk, especially when markets are thin. Hence, the value of a trader has to do with being around to mitigate this risk, being able to trade with others at key times. Concavity is key for us as individuals and societies are risk averse and hence care about this risk ex-ante. As a result, we show that our measure of financial centrality features risk aversion, prudence, and the coefficient of variation of income. We then identify and quantify value of key players in terms of whether they are likely to be able to smooth the resulting market participation risk and how valuable that smoothing would be when they are there.

The centralized planning problem that delivers Pareto optimal allocations is the problem of maximizing a Pareto weighted sum of ex-ante expected utilities subject to shock contingent resource and to market participation constraints. Financial centrality of an agent $i$ is then the increment in ex ante social value, an increase in the objective function of planner, derived from injecting liquidity to $i$ ex-ante - that is,

$$
F C_{i}:=\text { Marginal Social Value of giving } \epsilon>0 \text { to } i \text { whenever she can trade. }
$$

The first order conditions with respect to this liquidity epsilon, when $\epsilon$ is driven to zero, is then the value of liquidity and the correct measure of financial centrality of each trader $i$. It is, again, the expectation of the joint product of the value of liquidity as the shadow price in the resource constraint and the participation indicator of that player $i$.

We generalize the results to incorporate heterogeneity in ex-ante Pareto weights, heterogeneity in means and in variances of liquidity shocks, correlation across shocks, and differential risk aversion. We also allow all agents to see in advance an aggregate shock that contains information about both market participation and income risk. This serves as a reduced from way to capture that fact that the importance of a player can depends on aggregate market conditions. To summarize results in this more general context, the most financially central agents are those who trade often; are more likely to trade when there are few traders; more likely to trade when income risk is high, and income shocks are positively correlated; more like to trade when attitudes towards risk are more sensitive in the aggregate (high average risk aversion); more likely to trade with "distressed" institutions; and trade when there are tail risk, macro shocks which co-determine both income and risk characteristics but also limited participation -- a shock to cross sectional dispersion, spirit of Lehman type events.

We also consider a Walrasian, decentralized interpretation of our measure of financial centrality. In an ex-ante securities market, agents will buy and sell claims to receive and give 
income transfers contingent on subsequent market participation shocks and contingent on subsequent income draws for those in a market. In equilibrium prices of securities will be such that net excess demand for securities is zero. These Arrow Debreu securities are priced by the Lagrange multiplier on the resources constraint or, roughly, by the marginal utility of consumption. Thus we can determine the value of a bundled-security associated with the name of the trader $i$ as a security paying off one unit whenever $i$ is in the market. All agents can buy and sell these security bundles, and they have prices which correspond to the expected product of $i$ being in the market and the shadow price of resources at those times. Hence this is fully equivalent with the measure of financial centrality we described earlier.

More generally Pareto weights of traders can differ. The preceding discussion was normative: we had considered how a planner with given preferences should measure the relative importance of increasing the endowment of agents whenever they are able to trade. We then turn to an alternative, positive approach. We study whether in some feasible, standard solution concepts, agents with higher centrality get rewarded with higher consumption. In a model with CARA utility and Gaussian shocks, this translate into having a higher Pareto weight in the planner's representation of the consumption allocation. We show that if the solution concept is Nash bargaining, then there is a positive linear relationship between the Pareto weight of an agent in the planner's problem and her financial centrality measure. Similarly, with Kalai-Smorodinsky bargaining, we show that the representing Pareto weights depends on the aforementioned market thickness terms that affect financial centrality (such as market size when the trader is present, volatility, and so on).

We apply this perspective to the data. In an observational analysis, we investigate whether there is empirical content in our theoretical approach. Using the Townsend Thai village data with a panel over 15 years, we look at 338 households across 16 villages where we have detailed data on consumption, income, and transactions across villagers. We use whether a household has reported making or receiving a transfer to any other household in a given month as a measure of being active in the network in a given period. Our positive analysis under Nash or Kalai-Smorodinsky bargaining suggest that those who participate when the market is thin (few active trader, greater volatility) should receive higher Pareto weights in the planner's representation. We use the panel to estimate a household fixed-effect of consumption to estimate a monotonically increasing function of the Pareto weight. We then regress this fixed effect on variables that capture whether the market has few traders in months when the household is active and whether the market has more income volatility when the household is active. We show that, indeed, a one standard deviation increase in market thinness by either measure corresponds to roughly a 0.1 standard deviation increase in the consumption fixed effect, which is consistent with the theory.

For the most part, we take as given the exogenous stochastic process which operates through networks to generate market participation shocks. Is this sense our markets and the observed linked, networked structures are exogenous. We also make the assumption of independence between income shocks and market participation shocks, mainly for pedagogy, in order to 
separate two distinct effects that a liquidity injection may have on social welfare. However we show how we can make market participation endogenous, yet retain in the centrality formula the earlier results, but also add to the formula this new, endogeneity part. So there are two pieces. The first, which we dub the risk sharing effect, measures how an additional dollar given to agent $i$ propagates through the economy, taking the market participation process as given. The second effect, which we call the participation effect, measures how giving an additional dollar to agent $i$ changes the (endogenous) participation decision by all agents in the economy.

For the participation effect, there is a related literature. Chang and Zhang (2016) develops a dynamic model of network formation in over-the-counter markets in which traders choose with whom to connect as well as whether to remain active in each period. Traders with higher trading needs optimally choose to match with traders with lower needs for trade, and this endogenously generates a core-periphery market structure. Other papers with endogenous network formation include Babus (2016), Franklin et al. (2009), Hojman and Szeidl (2008), Gale and Kariv (2007) and Farboodi (2015). See Jackson (2008)for a review.

To understand the participation effect in our model in greater detail, consider the following pedagogical example. Suppose every individual first gets a signal about the income draws (both hers and of other agents in the economy) before income is realized. Then, given the signal, the agent decides whether or not to access the market at some fixed cost. The endogenous choices of the agents to participate will correlate participation with income draws. We show in a general environment with endogenous market participation, how to characterize financial centrality. It remains the same as with exogenous participation, plus an extra term. This extra term weights, by percent change in the market participation decision of each agent, the Pareto-weighted utilities of all agents. Essentially, taking endogenous participation into account makes an agent more central if the liquidity injection to the agent corresponds to an increase in participation configurations where more valuable agents enter the market.

The remainder of the paper is organized as follows. Section 2 presents the baseline environment. We define our notion of financial centrality in Section 3. Section 4 computes financial centrality under our core assumptions. We extend our environment in five ways, allowing for heterogeneous endowment distributions, heterogenous preferences, correlated market participation and income draws, and heterogenous Pareto weights in Section 5 and we compute financial centrality in each case. Section 6 then extends our environment to one in which agents endogenously choose to participate, as until now the model has maintained an exogenous participation process. We explore several stylized market formation processes in Section 7. In Section 8 turns to a positive analysis of the determination of Pareto weights in the planner's representation of the consumption allocation. We study Nash bargaining and Kalai-Smorodinsky bargaining solution concepts to show that agents with higher centrality get rewarded with higher consumption. We then turn to the Townsend Thai data to demonstrate (observational) patterns in the data that are consistent with our framework in Section 9. In Section 10, we study two extensions that depart from the centralized market perspective we have considered previously. 
First, we look at the case of having multiple segmented markets and second, we study financial centrality when the consumption is not allocated by the planner. Section 11 is a conclusion.

\section{ENVIRONMENT}

2.1. Setup. We consider an endowment economy with a set $I=\{1, \ldots, n\}$ of agents and one good. We study a one period economy, but this can be easily generalize to multiple periods. Agents face idiosyncratic income risk, where $y=\left(y_{1}, \ldots, y_{n}\right)$ denotes the vector of income realizations for all agents in the economy, which we assume are drawn from some distribution $F(y)$. In most applications, we will assume income draws are independent across agents, but this needs not be the case. Let $\mu_{i}=\mathbb{E}\left(y_{i}\right)$ and $\sigma_{i}^{2}=\mathbb{E}\left(y_{i}-\mu_{i}\right)^{2}$. Agents have expected utility preferences, with utility function $u_{i}\left(c_{i}\right)$, which we assume to be strictly increasing, strictly concave, and sufficiently smooth (i.e., all derivatives exist). We will also assume that $u^{\prime \prime \prime}(c)>0$, making agents prudent. Risk aversion can vary with wealth.

The leading example in this paper (as in much of the finance literature) is an environment with CARA preferences and Gaussian income shocks:

$$
u_{i}\left(c_{i}\right)=-r_{i}^{-1} \exp \left(-r_{i} c_{i}\right) \text { and } y \sim \mathcal{N}(\mu, \Sigma) .
$$

This parametrization will prove useful to obtain closed form expressions in Section 5.

The only point of departure with the usual risk sharing environment is on trading opportunities, or market participation. Not every agent is present in the market in every state; only a random set of agents gets access to the market, which can be thought of as a meeting place where they can trade. If agents don't have access to this market, they are in autarky and have to consume their endowment. ${ }^{1}$

Formally, let $\zeta \in\{0,1\}^{n}$ be the market participation vector, which we model as a shock to the consumption set of agents. That is, if $\zeta_{i}=0$ then $c_{i}=y_{i}$. However, if $\zeta_{i}=1$, then consumption and income do not have to coincide as agents can make transfers at such states. The relevant state, in the Arrow and Debreu sense of enumerating all shocks and indexing the commodity space by them, is then $s=(y, \zeta) \in S:=\mathbb{R}_{+}^{n} \times\{0,1\}^{n}$. A feasible consumption allocation is a function $c(s)=\left(c_{i}(s)\right)_{i \in I}$ such that, for every $s=(y, \zeta), c_{i}(s)=y_{i}$ whenever $\zeta_{i}=0$ and it is resource feasible: i.e., $\sum_{i} \zeta_{i} c_{i} \leq \sum_{i} \zeta_{i} y_{i}$ for all $s$.

State $s$ is drawn from a probability distribution $\mathrm{P}(y, \zeta)$ which is common knowledge among agents, and we assume it to be discrete for most proofs, for expositional simplicity. This is a primitive of our baseline environment, which models, in reduced form, the market participation process, be it as in classical search models (as in Duffie et al. (2005)), directed search (as in Armenter and Lester (2015)), or random matching (as in Kiyotaki and Wright (1989); Trejos and Wright (1995), etc). Our baseline model will assume that market participation is drawn independently from the income distribution (i.e., $y \perp \zeta$ ). However, we generalize this in Section 6 .

\footnotetext{
${ }^{1}$ In Section 10 we explore a model with several segmented markets, and show how to map all of the results of this special model to the general case.
} 
Suppose the optimum can be determined as if there were a "planner"who tries to chooses among resource feasible allocations to maximize a linear welfare functional, with Pareto weights vector $\lambda \in \mathbb{R}_{+}^{n}$, effectively choosing $c(s)$ to solve:

$$
V:=\max _{\left(c_{i}(\cdot)\right)_{i=1, \ldots, n}} \mathbb{E}_{s}\left\{\sum_{i=1}^{n} \lambda_{i} u_{i}\left[c_{i}(s)\right]\right\}
$$

subject to

$$
\sum_{i=1}^{n} \zeta_{i} c_{i}(s) \leq \sum_{i=1}^{n} \zeta_{i} y_{i}(s) \text { for all }(y, \zeta)
$$

and

$$
c_{i}(s)=y_{i} \text { for all } s=(y, \zeta): \zeta_{i}=0 .
$$

2.2. Discussion. Our basic environment is easily generalized, so the model is more applicable than might seem a priori. First of all, we can index by time, with long or even infinite horizon. We can entertain Markov process on shocks. Our timeline can be divided into sub-periods: traders meet in a market for two or more periods before the next market participation draw (and we allow both implementation via bilateral links of a multi-person outcome as well as borrowing and lending with risk contingencies within the longer period). We are featuring one good but we can easily generalize the notation and allow commodity vectors over goods. Then there would be a sequence of resource constraints (market clearing), one for each good; utility functions still strictly concave though. Likewise we can reinterpret goods as securities, endowments as portfolios, and derive the marginal liquidity valuations for each asset separately, one at time, for each trader $i$. Trivially, our setting could be partial equilibrium with prices of all goods, or assets, fixed outside, as in a small open economy, one market at a time, or one village at a time. In this case value functions would be strictly concave over a selected numeraire good, taking outside prices as given. Finally its easy to allow preference shocks rather than endowment shocks.

Though dynamics could easily be incorporated throughout most of the paper, we spare the reader the requisite notation. In particular we would have to keep track of histories when we endogenize market participation, in Section 6.

\section{Financial Centrality}

3.1. Centrality as the Marginal Value of Liquidity. We consider a thought experiment: increasing the endowment of a subset of agents $J \subseteq I$, across all values of income, whenever they can trade. That is, the policy consists of offering a "credit line" but really a transfer, contingent only on participation and without any repayment obligations,. More formally, $t=\left(t_{j}\right)_{j \in J} \geq 0$ changes the income process for agent $j \in J$ to $\hat{y}_{j}(s)=y_{j}+\zeta_{j} t_{j}$ for all $s=(\zeta, y)$. This is a commitment to a named trader $j$ without knowing what situation the trader will be in. The planner has $T>0$ units of consumption to finance these transfers. 
If $V(t)$ is the maximization problem's value function, with income process $y_{j}=\hat{y}_{j}$, the planner would choose $t=\left(t_{j}\right)_{j \in J} \geq 0$ to solve

$$
\max _{t \in \mathbb{R}_{+}^{\# J}} V(t) \text { s.t } \sum_{i \in J} t_{j} \leq T .
$$

Note that $V(t)$ here is a general value function, which could come from the corresponding to the solution $V$ of program (2.1), but this not required. We can define financial centrality generally in this way.

Definition 3.1. We define financial centrality of agent $i \in I$ as

$$
F C_{i}:=\left.\frac{\partial V(t)}{\partial t_{i}}\right|_{t=0}
$$

i.e., the increment in value for the planner of providing liquidity to agent $i$ whenever she can trade.

The next proposition is one of the main arguments to use financial centrality as the relevant relative importance measure of providing liquidity to each agent in the margin.

Proposition 3.1. Suppose $V(\cdot)$ is concave and differentiable at $t=0$. Moreover, suppose $\exists i \in J$ such that $F C_{i}>F C_{j}$ for all $j \in J \sim\{i\}$. Then, there exist $\hat{T}_{J}>0$ such that if $T \leq \hat{T}_{J}$ the unique solution $t^{*}=\left(t_{j}^{*}\right)_{j \in J}$ to program (3.1) is $t_{i}^{*}=T$ and $t_{j}^{*}=0$ for all $j \in J \sim\{i\}$.

The intuition behind Proposition 3.1 relies on the fact that if $V$ is differentiable at $t=0$, then it is approximately a linear function, and hence it is locally maximized by allocating all the resources to the agent with highest marginal value, given by our notion of financial centrality.

Proof. See Appendix A.

We next develop a useful formulation of financial centrality, in terms of the multipliers of the maximization problem in 2.1. Let $\widehat{q}(y, \zeta)$ be the Lagrange multiplier for the first condition, and define an auxiliary useful multiplier vector

$$
q(y, \zeta): \widehat{q}(y, \zeta):=q(y, \zeta) \mathrm{P}(y, \zeta),
$$

and let $\gamma_{i}(s)$ be the corresponding Lagrange multiplier for the non negativity constraint $c_{i} \geq 0$. The Lagrangian for 2.1 is then

$$
\mathcal{L}=\mathbb{E}_{s}\left\{\sum_{i \in I} \lambda_{i} u_{i}\left[c_{i}(s)\right]+q(s) \zeta_{i}\left[y_{i}-c_{i}(s)\right]+\gamma_{i}(s) c_{i}(s)\right\}
$$

In the baseline model, we assume market participation is independent from income draws. In this case, financial centrality can be expressed using the envelope theorem on program 2.1. 
Proposition 3.2. Suppose $\zeta \perp y$, and let $q(s)$ and $\gamma_{i}(s)$ be the multipliers of Lagrangian 3.3 for Program 2.1. If for all $i \in I$ and $s \in S$ we have $c_{i}(s)>0$ whenever $\zeta_{i}=1$, then

$$
F C_{i}=\mathbb{E}_{s}\left\{\zeta_{i} q(s)\right\}
$$

If not, then $F C_{i}=\mathbb{E}_{s}\left\{\zeta_{i}\left[q(s)+\gamma_{i}(s)\right]\right\}$

Proof. We use the classical envelope theorem on a variation of program 2.1, changing the income of agent $i$ to $\hat{y}_{i}=y_{i}+\zeta_{i} t_{i}$. Then, the envelope theorem implies

$$
\left.\frac{\partial V}{\partial t_{i}}\right|_{t=0}=\mathbb{E}_{s}\left[\zeta_{i} \frac{\partial \mathcal{L}}{\partial y_{i}} \times \frac{\partial y_{i}}{\partial t_{i}}\right]=\mathbb{E}_{s}\left\{\zeta_{i}\left[q(s)+\gamma_{i}(s)\right]\right\} .
$$

If $c_{i}(s)>0$ for almost all $s$, then complementary slackness implies $\gamma_{i}(s)=0$ for almost all $s$, and hence $\mathbb{E}_{s}\left\{\zeta_{i} \gamma_{i}(s)\right\}=0$, proving the desired result.

The multiplier $q(s)$ is, of course, the marginal value of consumption, at the (constrained) efficient allocation $c_{i}(\cdot)$. As we will see below, when defining a Walrasian equilibrium in an Arrow Debreu economy defined on this environment, $q(s)$ will correspond to the equilibrium price of the Arrow Debreu security that pays only at state $s$. As such, equation 3.4 is effectively the price of a fictitious asset that pays 1 consumption unit whenever $\zeta_{i}=1$, using $q(s)$ as its pricing kernel.

As we noted above, $V(\cdot)$ need not only reflect the Program 2.1. Consider a modification where we have constrained efficiency. So we add to Program 2.1 some additional constraint , that

$$
\Xi(c, y, \zeta) \leq 0 \text { for all }(y, \zeta): \zeta_{i}=1 .
$$

This function represents some (arbitrary) frictions in consumption allocations and transfers. For instance it could represent constraints as to who is able to make (how much) transfers to whom. In this case, financial centrality would be

$$
F C_{i}=\mathbb{E}_{s}\left\{\zeta_{i}[q(s)]+p(s) \cdot \frac{\partial \Xi(s)}{\partial y_{i}}\right\},
$$

where $p(s)$ is the Lagrange multiplier of the friction constraints.

\subsection{Decentralization as Arrow Debreu Economies with Exogenous Market Partic-}

ipation. In this section, we introduce a natural candidate for implementation of the Planners optimal consumption allocation: a Walrasian equilibrium with transfers. We present the Second Welfare Theorem for this setting, showing that any constrained Pareto optimal allocation can be implemented by one such equilibria (with suitably chosen lump sum transfers) and we show how equilibrium prices for Arrow Debreu securities correspond to the shadow values of resource constraints in the planner's problem (a classical result in implementation theory). As a side product, the proof gives us a different interpretation to our centrality measure; namely, the financial centrality of agent $i$ would be the price (on the implementing Walrasian Equilibrium with transfers) of an asset that pays off only when agent $i$ has market access . 
We consider an Arrow Debreu economy, where agents can buy and sell claims on income and consumption, contingent on the configuration of the market and the nature of income shocks. However, agents cannot buy or sell income claims that will pay off at states where they are unable to trade (since there is no physical way to make such transfers), which we formalize as as "consumption space shocks" (as in Mas-Colell et al. (1995)). Formally, let $A_{\hat{s}}$ denote the Arrow Debreu (AD) asset $^{2}$ that pays 1 unit of the consumption good if the state is $s=\hat{s}$, and nothing if $s \neq \hat{s}$., and $a_{i}(\hat{s}) \in \mathbb{R}$ the demand of asset $A_{\hat{s}=s}$ by agent $i$. Consumption for agent $i$ at state $s=(y, \zeta)$ is then $c_{i}(s)=y_{i}+a_{i}(s)$. The market participation constraint can be introduced by imposing a physical constraint: whenever $\zeta_{i}=0$ we must have $a_{i}(s) \in\{0\}$ (i.e. agents cannot trade in assets that they will not be able to be present in the market to clear the trades ex-post).

To simplify proofs and exposition, we consider cases where there is only a countable number of possible income shocks, so that $S=\prod_{i}\left(Y_{i} \times\{0,1\}\right)$ is also countable, and where $\mathrm{P}(s \in S)>$ 0 for all $s \in S$. Given an Arrow Debreu prices $\hat{r}(s)$ for each $A_{s}$, and a vector of transfers $\tau=\left(\tau_{i}\right)_{i \in I}$ such that $\sum_{i \in I} \tau_{i}=0$, agents choose consumption and asset purchases to maximize expected utility, given her budget constraint:

$$
\begin{gathered}
\max _{\left\{c_{i}(s), a_{i}(s)\right\}} \mathbb{E}_{s}\left\{u_{i}\left[c_{i}(s)\right]\right\} \\
\text { s.t }: \begin{cases}c_{i}(s)=y_{i}(s)+a_{i}(s) & \text { for all } s \in S \\
a_{i}(s)=0 & \text { for all } s \in S: \zeta_{i}=0 \\
\sum_{s \in S} a_{i}(s) \hat{r}(s) \leq \tau_{i} . & \end{cases}
\end{gathered}
$$

As we did when defining the Lagrange multipliers for the planning problem, we normalize the price function as $r(s)=\hat{r}(s) / \mathrm{P}(s)$, changing the budget constraint in the consumer problem as

$$
\mathbb{E}_{s}\left[a_{i}(s) r(s)\right]:=\sum_{s \in S} a_{i}(s) r(s) \mathrm{P}(s) \leq \tau_{i} .
$$

A Walrasian equilibrium with transfers $\tau$ is a triple $(c, a, r)=\left(\left\{c_{i}(s), a_{i}(s)\right\}_{i \in I, s \in S},\{r(s)\}_{s \in S}\right)$ such that

- $\left\{c_{i}(s), a_{i}(s)\right\}_{s \in S}$ solves 3.5 with budget constraint 3.7 for all $i \in I$, given (normalized) prices $r(s)=\hat{r}(s) / \mathrm{P}(s)$ and transfers $\tau=\left(\tau_{i}\right)_{i \in[n]}$,

- asset markets clear: $\sum_{i \in I} a_{i}(s)=0$ for all $s \in S,{ }^{3}$ and

- consumption good markets clear: $\sum_{i \in I} \zeta_{i} c_{i}(s) \leq \sum_{i \in I} \zeta_{i} y_{i}$ for all $s \in S$.

A Walrasian Equilibrium is an equilibrium $(c, r)$ with no transfers $(\tau=0)$. In Proposition 3.3 below, we show a version of the First and Second Welfare Theorems for this economy, which is

\footnotetext{
${ }^{2}$ Formally, $A_{\hat{s}}(s)=\left\{\begin{array}{ll}1 & \text { if } s=\hat{s} \\ 0 & \text { otherwise }\end{array}\right.$ is the return matrix of the AD security paying only at state $\hat{s}$

${ }^{3}$ If $\exists \tilde{s} \in S: \mathrm{P}(\tilde{s})=0$, then we can interpret this condition as imposing the constraint that $a_{i}(\tilde{s})=0$ for all $i \in I$ (i.e. agents cannot trade in probability zero events)
} 
just an application of the classical welfare theorems to this environment (See Mas-Colell et al. (1995)). This can be qualified as a welfare theorem with "constrained efficiency", since the constraint that lack of market access (i.e. $\zeta_{i}=0$ ) implies autarkic consumption is interpreted as a physical constraint (i.e., a social planner could not change an inactive agent's consumption either).

Proposition 3.3 (Welfare Theorems). Suppose $\zeta \perp y$. Take a Planners problem 2.1 with Pareto Weights $\lambda \in \Delta^{I}$, and an optimizing allocation $c=\left(c_{i}(s)\right)_{i \in I, s \in S}$, with normalized Lagrange multipliers $q(s)$ (as defined in 3.2). Then, $(c, r)$ is a Walrasian Equilibrium with Transfers $\tau$, where $r(s)=q(s)$ for all $s \in S$ and $\tau_{i}=\mathbb{E}_{s}\left\{\left[c_{i}-y_{i}(s)\right] q(s)\right\}$. On the other hand, if $(c, r)$ is such an equilibrium with transfers $\tau$, then $\exists$ Pareto Weights $\lambda \in \Delta^{I}$ such that $c$ is the allocation solving Planners problem 2.1 (where we again have $q(s)=r(s)$ ).

Proof. See Appendix A.

An important Corollary of Proposition 3.3 (and most classical proofs of Second Welfare Theorems in various settings) is that it gives us an explicit formulation for the equilibrium AD security prices at the implementing equilibrium, which coincide with the shadow values $q(s)$ at the resource constraint at each state $s$. But then, since we can interpret this economy as one with complete markets (once we interpret market participation shocks as consumption sets shocks) $r(s) \mathrm{P}(s)$ is a pricing kernel, which greatly simplifies the pricing of additional assets, if available to the market. More explicitly, if we add to this economy, on top of the AD securities offered, an asset with return payoff function $\rho(s) \in \mathbb{R}$, its (no arbitrage) equilibrium price in this economy would be

$$
\text { Price }=\mathbb{E}_{s}[\rho(s) \times r(s)]:=\sum_{s \in S} \rho(s) r(s) \mathrm{P}(s) .
$$

Using the results from 3.3, we can then show that financial centrality can be thought as the equilibrium price of an asset (which we dubbed personalized debt) with return payoff matrix $\rho^{i}(s)=1$ if $s: \zeta_{i}=1$.

Corollary 3.1. Suppose $y \perp \zeta$ and let $(c, r)$ be the Walrasian equilibrium with transfers $\tau=$ $\left(\tau_{i}\right)_{i \in I}$ that implements the Planner's Problem 2.1 optimal allocation $c$ with Pareto weights $\lambda \in \Delta$. Then

$$
F C_{i}(\lambda)=\sum_{s \in S} \rho^{i}(s) \hat{r}(s)=\sum_{s \in S} \rho^{i}(s) r(s) \mathrm{P}(s) .
$$

That is, financial centrality is the price of a personalized debt asset implementing Walrasian Equilibrium with transfers.

Of course, there is a mapping between a Walrasian Equilibrium without transfers and its corresponding utilitarian planner representation, with its Pareto weight vector $\lambda$. Two special cases are of interest.

In the benchmark case of CARA preferences and Gaussian income draws, assuming constrained efficient allocations are implemented without lump sum transfers, we obtain a fixed 
point equation mapping the primitives of the model (income distribution moments and preferences) to the Pareto weights of the Planner's Problem which we derive in Appendix B.

We also show that in the case where the Planner has uniform Pareto weights (i.e., $\lambda_{i}=1 / n$ for all $i$ ), preferences are identical and shocks are i.i.d Gaussian variables, then the Planner's problem can be implemented by a Walrasian Equilibrium with no transfers with $q(s)=$ $\exp \left(-r \bar{y}_{\zeta}\right)$ and $c_{i}(s)=\zeta_{i} \bar{y}_{\zeta}+\left(1-\zeta_{i}\right) y_{i}$, where $\bar{y}_{\zeta}:=\frac{1}{n_{\zeta}} \sum_{j \in[n]} \zeta_{j} y_{j}$ is the mean income of agents in the market, and $n_{\zeta}:=\sum_{j \in I} \zeta_{j}$ is the market size at state $s$. Moreover, the price of personalized debt is simply $F C_{i}=\mathbb{E}_{\zeta}\left\{\zeta_{i} \exp \left(\gamma \frac{\sigma^{2}}{2}\right)\right\}$.

3.3. Market Participation within a Network. An important object in our environment is the vector of market participation shocks. We have modeled it with some reduced form distribution $\mathrm{P}(\zeta)$, which we take as a fundamental of the model. Our main objective is to provide, for any such a participation process, a measure of financial centrality for a planner tasked with maximizing social welfare. We now investigate how can we map data on trades and social and financial networks, into this environment.

In many contexts researchers and policymakers have data on economic networks. One approach consists of leveraging this and modeling the market participation process as a function of the network data available:

$$
\zeta \sim F(\cdot \mid \text { Network }) .
$$

The network generates parameters that are fundamental to the market "matching" process. For example, suppose one and only one trader in the network is chosen as a seed. For simplicity say everyone chosen with equal probability. Next, once a seed is specified, there is a probability distribution governed by the graph as to which traders will show up in the market match. One can think of this as if invitations are passed stochastically through the network, for instance, starting from the seed. Of course this allows special cases in which these seed probabilities are trader specific, or even one of the agents could always be the seed, as if deterministic.

The data available depends on the application. The data may include the invitation process itself, i.e. who was the seed and who was contacted. Alternatively, we may only see outcomes in terms of time varying participation in the market. These considerations matter for identification and parameterization of particular structures, if we see the process. Otherwise, one can use the histogram of market participation as the fundamental.

We give two distinct examples. The first example is as described in Hendershott and Madhavan (2015) in CDS securities in corporate bonds. It is neither completely centralized nor entirely bilateral. At a point in time a trader (our seed) has a need to change its financial position and initiates a request for trade, posting on a financial platform an invitation for specified other named individuals to participate in an auction of limited duration. These messages may or may not capture the attention of their targets, depending on what they are doing at the time, i.e. paying attention to the monitor or not. Equivalently, the initiator may or may not be in a good position, in terms of her ability to attract traders. There are various versions of these technologies, becoming increasing common and blurring the distinction between OTC 
and centralized markets. For example one of distinguishing factors in Market Axess is the inclusion of a large base of dealers, investment managers and alternative market participants in the same trading pool. Tradeweb Markets builds and operates electronic over-the-counter marketplaces with voice, electronic, and hybrid platforms.

The second example is described by Li and Schürhoff (2014) for municipal bonds, and it appears at first blush to allow bilateral links, only, but this impression is misleading, which is why we feature it here. The data source is the proprietary Transaction Reporting System audit trail from the MSRB, municipal bond transactions, over 15-year period from February 1998 to December 2012. There are identifiers for the dealer firms intermediating each trade and also for customer trades, in which case the data identify the dealer buying and the dealer selling the bond. Municipal bond dealers intermediate round-trip trades not only by taking the bond into inventory for a time but rather asking the seller to wait until a matching buyer is found. In an example round-trip transaction, an investor sells bonds to a dealer and then the dealer sells the same bonds to another investor or other dealers. Thus there are intermediation chains consistent of these bilateral links, and these can extend up to seven dealers .

In this sense the bilateral model is not as restrictive as it seems. That is, our multilateral market model can be implemented as a series of bilateral trades. As in Allen and Gale (2000), Alvarez and Barlevy (2015), suppose a "transaction chain" is realized:

$$
i_{1} \underset{\text { trades with }}{i_{2}} \text { trades with }^{\longleftrightarrow} \underset{\text { trades with }}{\longleftrightarrow} i_{k}
$$

That is agents transfers $t_{i, i+1}$ and $t_{i+1, i}$. and the net transfers . With decentralized agreement up front, agents only need to know they are currently paired and the consensus, the overall target consumption. See Appendix C for proofs and a more detailed exposition.

Much in this spirit is Ambrus et al. (2017), which considers the effect of local information constraints in risk-sharing networks. They assume individuals only observe the endowment realizations of their neighbors, and bilateral risk-sharing arrangements can only depend on this commonly observed information. They derive necessary and sufficient conditions for Pareto efficiency under these constraints, and provide an explicit characterization of Pareto efficient arrangements under CARA utilities and normally distributed endowments. With independent endowments, a local equal sharing rule is shown to be optimal.

\section{Computing Financial Centrality: A Baseline Case}

In this section, we continue to assume that income realization and market participation shocks are independent. We relax this in Sections 5 and 6 . Under this assumption, the financial centrality measure can be further decomposed as

$$
F C_{i}=\mathbb{E}_{\zeta}\left\{\zeta_{i} \mathbb{E}_{y}[q(y, \zeta) \mid \zeta]\right\}=\mathbb{E}_{\zeta}\left\{\zeta_{i} \times \mathbb{E}_{y}[q(y, \zeta)]\right\}
$$

This is particularly useful in a number of cases, where market fundamentals depend only on aggregate statistics of market size and volatility, rather than the identities of the agents trading. 
We will assume, throughout the rest of the paper, that $\lim _{c \rightarrow 0} u_{i}^{\prime}(c)=+\infty$ for all $i \in I$, so we always have $c_{i}(s)>0$ whenever $\zeta_{i}=1$ and $\sum \zeta_{j} y_{j}>0$.

The main benchmark we consider is one with homogeneous preferences, independent and identically distributed income draws, and an utilitarian planner. Such an environment has all agents being virtually identical in almost all aspects, except their relative positions through market participation shocks $\zeta$. If agents have different financial centrality in this setting, it can only come from their heterogeneity in their positions in the financial network, which is the new dimension we introduce in this paper.

Formally, we assume $u_{i}(\cdot)=u(\cdot)$ for all $i \in I$ and $\left\{y_{i}\right\}$ are independent and identically distributed where $\mathbb{E}_{y}(y)=\mu$ and $\operatorname{var}(y)=\sigma^{2}$. Our aim will be to get a closed form approximation to $q(s)$. Two quantities will become particularly important in our analysis: the number of agents that are able to trade and the average income of agents that are able to trade. Therefore let

$$
n_{\zeta}:=\sum_{i=1}^{n} \zeta_{i}
$$

denote the number of agents that are able to trade and

$$
\bar{y}(s):=\frac{1}{n_{\zeta}} \sum_{i=1}^{n} \zeta_{i} y_{i}
$$

as the average income of agents able to trade. In this very simple case, we get the following approximation.

Proposition 4.1. Suppose $u_{i}=u$ and $\lambda_{i}=1 / n$ for all $i$, and income draws are independent and identically distributed across agents. Then $q(s)=u^{\prime}[\bar{y}(s)], c_{i}(s)=\zeta_{i} \bar{y}(s)+\left(1-\zeta_{i}\right) y_{i}$. Moreover, if $u$ is analytic (which imply Taylor expansions are valid) then we can approximate

$$
F C_{i} \approx \mathbb{E}_{\zeta}\left[\zeta_{i} u^{\prime}(\mu)\left(1+\gamma \frac{\sigma^{2}}{n_{\zeta}}\right)\right]
$$

where $\gamma=(1 / 2) u^{\prime \prime \prime}(\mu) / u^{\prime}(\mu)$.

The approximation of financial centrality given in Proposition 4.1 gives us a summary of the relevant moments of the market participation process, for the purpose of calculating financial centrality. Note that we can rewrite equation 4.1 as

$$
F C_{i} \approx u^{\prime}(\mu) \mathrm{P}\left(\zeta_{i}=1\right) \times\left[1+\gamma \sigma^{2} \mathbb{E}\left(\frac{1}{n_{\zeta}} \mid \zeta_{i}=1\right)\right] .
$$

What matters here is the variance of mean income, which is the relation between income volatility $\left(\sigma^{2}\right)$ and market size $n(\zeta)$, giving us the only relevant moment of the distribution of $\zeta$ and income volatility $\sigma^{2}$.

Proof. The first order conditions of Program 2.1 with Lagrangian defined in 3.3 with respect to $c_{i}(s)$ whenever $\zeta_{i}=1$ is $\lambda_{i} u_{i}^{\prime}\left[c_{i}(s)\right]=q(s)$ (without taking into account the non-negativity constraint over consumption). Therefore, if $\lambda_{i}=\lambda_{j}=1 / n$ for all $i, j \in[n]$ and $u_{i}=u$ for all $i$, 
we then get that if $\zeta_{i}=\zeta_{j}=1$ then $c_{i}(s)=c_{j}(s)$ (i.e. all agents participating in the market have equal consumption). Therefore, using the resource constraint, we obtain $c_{i}(s)=\bar{y}(s)$ whenever $\zeta_{i}=1$, and obviously $c_{i}(s)=y_{i}$ otherwise. The first order condition also implies then that $q(s)=u^{\prime}[\bar{y}(s)]$.

To obtain the approximation, we provide a heuristic proof: we first make a second order Taylor approximation $g(y):=u^{\prime}(y)$ around $y=\mathbb{E}(y)=\mu$ :

$$
u^{\prime}(\bar{y}) \approx u^{\prime}(\mu)+u^{\prime \prime}(\mu)(\bar{y}-\mu)+\frac{u^{\prime \prime \prime}(\mu)}{2}(\bar{y}-\mu)^{2}
$$

and then take expectations, we have

$\mathbb{E}\left[u^{\prime}(\bar{y}(s)) \mid \zeta\right] \approx u^{\prime}(\mu)+u^{\prime \prime}(\mu) \mathbb{E}(\bar{y}-\mu \mid \zeta)+\frac{1}{2} u^{\prime \prime \prime}(\mu) \mathbb{E}\left[(\bar{y}-\mu)^{2} \mid \zeta\right]=u^{\prime}(\mu)+\frac{1}{2} u^{\prime \prime \prime}(\mu) \sigma^{2} / n(\zeta)$,

using the facts that $\mathbb{E}(\bar{y})=\mu$ and that $\mathbb{E}(\bar{y}-\mu)^{2}=\sigma^{2} / n(\zeta)$ if income draws are i.i.d. Reorganizing this expression, we get the desired result.

This shows that centrality can be decomposed into two pieces. Financial centrality is higher when (1) the agent has a higher probability of trading $\left(\mathrm{P}\left(\zeta_{i}=1\right) \uparrow\right)$ and $(2)$ the market size conditional on the agent entering is smaller. Finally, the degree to which each of these matters can depend on the mean income, degree of risk aversion, degree of prudence (convexity of marginal utility of consumption, which governs precautionary savings), and variability of income (measured by the coefficient of variation). For example with $\log$ utility $\gamma=\frac{1}{\mu^{2}}$, with CES utility $u=\frac{c^{1-\rho}-1}{1-\rho}$ we get $\gamma=\frac{\rho(1+\rho)}{2 \mu^{2}}$, and with CARA preferences we have $\gamma=\frac{r^{2}}{2}$. As a technical aside, Taylor expansions around the mean are not always valid. See Loistl (1976) and Levy and Markovitz (1979) for a discussion. For example, if $u=-r^{-1} \exp (-r c)$, the approximation at $y=\mu$ is always valid.

In the particular example of the CARA and Normal model, we get an exact expression for financial centrality. Under homogeneity of preferences and an utilitarian planner, we have $c_{i}(s)=\bar{y}(s)$ whenever $\zeta_{i}=1$, and $q(s)=u^{\prime}[\bar{y}(s)]=\exp [-r \bar{y}(s)]$. Since $y_{i} \sim_{i . i . d} \mathcal{N}\left(\mu, \sigma^{2}\right)$ we have that $\bar{y}(s) \mid \zeta \sim \mathcal{N}\left(\mu, \sigma^{2} / n(\zeta)\right)$. Therefore

$$
\begin{aligned}
\mathbb{E}\left[\zeta_{i} q(s)\right] & =\mathbb{E}_{y, \zeta}\left\{\zeta_{i} \exp [-r \bar{y}(s)]\right\}=\mathbb{E}_{\zeta}\left\{\zeta_{i} \exp \left(-r \mu+\frac{r^{2}}{2} \frac{\sigma^{2}}{n(\zeta)}\right)\right\} \\
& =\underbrace{\exp (-r \mu)}_{u^{\prime}(\mu)} \mathbb{E}_{\zeta}\left\{\zeta_{i} \exp \left[\gamma \frac{\sigma^{2}}{n(\zeta)}\right]\right\}
\end{aligned}
$$

where, as we saw before, $\gamma=u^{\prime \prime \prime}(\mu) / 2 u^{\prime}(\mu)=r^{2} / 2$.

\section{Financial Centrality in More General Environments}

In this section, we develop expressions for financial centrality in more general environments. First, we add agent-specific heterogeneity in volatility. Second, we add heterogeneity in Pareto weights in the planner's problem. Third, we add heterogeneity in agent-specific income. Fourth, we relax the common preferences assumption. And fifth, we allow income 
draws to be correlated with market participation via shocks to fundamentals. In each of these cases we derive the formula for financial centrality and call attention to the changes, if any, to our core characterization. The basic formulation in each case stays the same, with minor changes per relaxation, as we add them sequentially. Proofs of all statements can be found in the appendix.

5.1. Volatility. Our first extension adds agent-specific heterogeneity. We suppose agents' income distributions have a common mean $\mu$ and idiosyncratic variance that is possibly correlated $\operatorname{var}\left(y_{1}, y_{2}, \ldots, y_{n}\right)=\Sigma_{n \times n}$. In this case let us define the average variance in the market $\bar{\sigma}_{\zeta}^{2}:=\frac{1}{n_{\zeta}} \sum_{i, j} \zeta_{i} \zeta_{j} \sigma_{i, j}$

We show that

$$
F C_{i} \approx \mathbb{E}_{\zeta}\left\{\zeta_{i} u^{\prime}(\mu)\left(1+\gamma \times \frac{\bar{\sigma}_{\zeta}^{2}}{n_{\zeta}}\right)\right\} .
$$

What this implies is that agents are more central if they are more likely to trade when (1) income volatilities are higher $\left(\sigma_{i}^{2} \uparrow\right)$ and $(2)$ trading agents have positively correlated income shocks $\left(\sigma_{i j}>0\right)$.

To see the intuition, consider for example a risk sharing model where agents can only meet pairwise. If the planner was able to choose the meeting distribution, he would pair agents with $\sigma_{i j}<0$ to smooth the income shocks. If $\sigma_{i j}>0$ then both agents do very well or poorly, exacerbating risk. Thus agents that are present whenever this happens are more valuable.

5.2. Pareto weights. Our next extension maintains the heterogeneity in volatility from before, but now introduces heterogeneity in Pareto weights. To make things simple consider either CES preferences, $u(c)=c^{1-\rho}-1 / 1-\rho$, or CARA preferences: $u(c)=-\frac{1}{r} \exp (-r c)$.

We show that

$$
F C_{i} \approx \mathbb{E}_{\zeta}\left\{\zeta_{i} u^{\prime}(\mu) \times \bar{\lambda}_{\zeta} \times\left(1+\gamma \frac{\bar{\sigma}_{\zeta}^{2}}{n_{\zeta}}\right)\right\},
$$

where the new term is now the average Pareto weight of trading agents.

In the CARA case, $\bar{\lambda}_{\zeta}:=\left(\prod_{i} \lambda_{i}^{\zeta_{i}}\right)^{1 / n_{\zeta}}$ is the geometric mean of the Pareto weights of agents in the market. In the CES case, $\bar{\lambda}_{\zeta}:=\left(\frac{1}{n_{\zeta}} \sum_{i} \zeta_{i} \lambda_{i}^{1 / \rho}\right)^{\rho}$ is the $L^{p}$-mean (or Dixit-Stiglitz aggregator) of Pareto weights. In either case, the main result is that agents who are there when the average agent is more important to the planner are more central, which is intuitive.

5.3. Expected income. Now we turn to the case where will introduce heterogeneity in expected income as well. We will maintain our previous extensions. To make things tractable, let us consider homogeneous CARA preferences, with $u(c)=-\frac{1}{r} \exp (-r c)$ and we assume that $y \sim \mathcal{N}(\mu, \Sigma)$, with $\Sigma$ a symmetric, positive definite variance-covariance matrix. 
In this case we can write

$$
F C_{i}=\mathbb{E}_{\zeta}\left\{\zeta_{i} \times \exp \left(-r \bar{\mu}_{\zeta}\right) \times \bar{\lambda}_{\zeta} \times \exp \left(\gamma \times \frac{\bar{\sigma}_{\zeta}^{2}}{n_{\zeta}}\right)\right\}
$$

where $\bar{\mu}_{\zeta}:=\frac{1}{n_{\zeta}} \sum_{i} \zeta_{i} \mu_{i}$ is the average income of trading agents. This extension teaches us that agents are more central if the average agent in the market has a lower endowment in expectation when the agent in question is in the market.

5.4. Preferences. We take our previous assumptions and add to this preference heterogeneity, with $u_{i}(c)=-r_{i}^{-1} \exp \left(-r_{i} c\right)$. It is useful to write $\bar{r}_{\zeta}:=\left(\frac{1}{n_{\zeta}} \sum_{i: \zeta_{i}=1} \frac{1}{r_{i}}\right)^{-1}$ and $\hat{\lambda}_{\zeta}:=\left(\prod_{i: \zeta_{i}=1} \lambda_{i}^{\bar{r}_{\zeta} / r_{i}}\right)^{\frac{1}{n_{\zeta}}}$. In this case the formulation becomes

$$
F C_{i}=\mathbb{E}_{\zeta}\left\{\zeta_{i} \times \exp \left(-\bar{r}_{\zeta} \bar{\mu}_{\zeta}\right) \times \hat{\lambda}_{\zeta} \times \exp \left(\frac{\bar{r}_{\zeta}^{2}}{2} \times \frac{\bar{\sigma}_{\zeta}^{2}}{n_{\zeta}}\right)\right\} .
$$

Again, we see that agents are more central if the degree of risk aversion when the agent is in the market is higher, which once again captures a sort of generalized notion of market thinness.

5.5. Aggregate shocks to fundamentals. Throughout this section and the paper thus far, we have assumed that income is orthogonal to market participation. We now relax this assumption. Assume that agents observe shocks to income volatility and expected income to certain agents. We maintain CARA utility as before with heterogenous risk aversion parameters and a jointly normal income distribution with heterogeneous mean, variance, and covarying income draws.

To begin with, there is an aggregate (fundamental) shock $z \in Z$ with some distribution $G(z)$. This fundamental shock affects preferences expected income $\mu(z)$, income variance $\Sigma(z)$, preferences $u_{i}(c, z)=-\frac{1}{r_{i}(z)} \exp \left(-r_{i}(z) c\right)$, and even the Social Planner's preferences $\lambda(z)$.

Income is modeled as $y=\mu(z)+\Sigma(z) x$, where $x \perp(z, \zeta)$ and is such that $x_{i} \sim_{i . i . d}$ $N(0,1)$. Market participation can be drawn as $\zeta \mid z \sim F(\zeta \mid \mu(z), \Sigma(z), r(z), \lambda(z))$ With these assumptions, income is Gaussian, and $(y \mid z) \perp(\zeta \mid z)$. The conditional independence will buy us a simple characterization.

The generalized version of centrality is

$$
F C_{i}=\mathbb{E}_{z, \zeta}\left\{\zeta_{i} \times \exp \left[-\bar{r}_{\zeta}(z) \bar{\mu}_{\zeta}(z)\right] \times \bar{\lambda}_{\zeta}(z) \times \exp \left[\frac{\bar{r}_{\zeta}^{2}(z)}{2} \times \frac{\bar{\sigma}_{\zeta}^{2}(z)}{n_{\zeta}}\right]\right\}
$$

where the market averages $\left(\bar{r}_{\zeta}, \bar{\mu}_{\zeta}, \bar{\lambda}_{\zeta}, \bar{\sigma}_{\zeta}^{2}\right)$ are functions of market fundamentals $z \in Z$. As expected, the intuitions from the previous exercises remain, now operationalized through the co-movements between $\zeta_{i}$ and $z$. 


\section{Endogenous Participation}

Until now the participation has been exogenously determined. We relax that assumption in this section, allowing agents' choices to determine whether or not they participate. This not only correlates $y$ and $\zeta$ through this endogenous decision making process, but changes the financial centrality expression as well. We characterize financial centrality in an extremely general form that works when there is heterogeneity in Pareto weights, preferences, and income distributions (as before) but also works when there is a causal link between income draws and market participation. There is an extra term, captured by the (endogenously determined) score function that captures how the probability of showing up to trade responds to the liquidity injection policy, which we refer to as the trade subsidy effect: how marginally increasing income in states that the agent trades in, increases both the likelihood that the agent trades and the concurrent market participation decisions of other agents, as well as how this increases welfare.

The pedagogical example we provide is one with private information and endogenous market participation. Consider a simple situation where every individual first gets a signal about the income draws (both hers and of other agents in the economy) before they are realized. After receiving this signal, the agent decides whether or not to access the market at fixed cost, assuming that, in equilibrium, agents have common knowledge both of every agents' equilibrium market participation decision and of the consumption allocation (as a function of the realized state). We can clearly see that the endogenous choices of the agents to participate end up correlating $y$ and $\zeta$, since market participation is a function of the private information agents have (about $y$ ) and this information is obviously correlated with income draws. Throughout this section, we will focus on the case of discrete income shocks, $Y$.

6.1. Example: Private Information and Costly Market Access. In our example, the consumption allocation $c=\left(c_{i}(s)\right)_{i \in I, s \in S}$ is common knowledge, but agents can only observe (objective) private information about both income shocks $y \in \mathbb{R}^{n}$, and about other agents information. We encode beliefs and higher order beliefs about income shocks and information using a type space structure, a modeling device introduced by Harsanyi (1967). Formally, we model agents' beliefs with a signal structure (or a common prior type space) $\mathcal{Z}=\left\{\left(Z_{i}, \beta_{i}: Z_{i} \rightarrow \Delta\left(Y \times Z_{-i}\right)\right)_{i \in I}, \beta_{0}\right\}$ where $z_{i} \in Z_{i}$ is the agent's signal (or type). Here this represents the information she observes observes before observing the draw of $s=(y, \zeta)$. $\beta_{0} \in \Delta\left(Y \times \prod_{i} Z_{i}\right)$ is a common prior distribution over income shocks and signals and $\beta_{i}\left(\cdot \mid z_{i}\right)$ is the conditional belief distribution over income shocks and signals of other agents, derived from $\beta_{0}$ using Bayes rule. ${ }^{4}$ Because $Y$ is assumed to be finite and the choice set for every agent is binary, we can focus also only on finite signal spaces. We also add the constraint that $\operatorname{marg}_{Y} \beta_{0}=F$ (i.e., the marginal distribution over income shocks coincide with the true distribution of shocks). Based on its type, agent $i$ decides whether or not to access the market at some disutility cost, $\kappa_{i}>0$.

$\overline{{ }^{4} \text { That is, for all }}\left(y, z_{i}, z_{-i}\right)$ we have $\beta_{i}\left(y, z_{-i} \mid z_{i}\right)=\frac{\beta_{0}\left[y,\left(z_{i}, z-i\right)\right]}{\sum_{\hat{t}_{i}} \beta_{0}\left[y,\left(\hat{z}_{i}, z_{-i}\right)\right]}$. 
The timing is as follows:

(1) Income shocks $y \in \mathbb{R}_{+}^{n}$ is drawn according to $F(y)$.

(2) Agents observe only $z_{i} \in Z_{i}$, which are jointly drawn with probability

$$
\mathrm{P}(z \mid y)=\beta_{0}(y, z) / \sum_{\hat{y} \in Y} \beta_{0}(\hat{y}, z) .
$$

(3) Agents decide whether to access the market $\left(\zeta_{i}=1\right)$ or not, given their private information $z_{i} \in Z_{i}$.

(4) State $s=(y, \zeta)$ is publicly observed, and agents consume according to allocation $c(s)$.

To characterize the agents' market participation decisions, they need to form beliefs over the vector of income draws and market participations. We will model this as a game, where agent's strategies are the mappings from information to market participation. The natural solution concept here is the Bayesian Nash Equilibrium (BNE): a profile of functions $\zeta_{i}^{*}: Z_{i} \rightarrow\{0,1\}^{5}$ is a BNE if and only if, for all $i \in I$ and all $z_{i} \in Z_{i}$

$$
\text { if } \zeta_{i}^{*}\left(z_{i}\right)=1 \Longrightarrow \mathbb{E}_{s}\left\{u_{i}\left[c_{i}(s)\right] \mid \zeta_{i}=1, z_{i}\right\}-\kappa_{i} \geq \mathbb{E}_{s}\left\{u_{i}\left(y_{i}\right) \mid z_{i}\right\}
$$

where the expectations for each agent is taken with respect to the probability measure

$$
\mathrm{P}\left(s=(y, \zeta) \mid z_{i}\right):=\sum_{y \in Y} \sum_{j \neq i}\left[\sum_{z_{j} \in Z_{j}: \zeta_{j}^{*}\left(z_{j}\right)=\zeta_{j}} \beta_{i}\left(y, z_{-i} \mid z_{i}\right)\right] .
$$

Given a signal structure $\mathcal{Z}$ and a BNE profile $\zeta^{*}=\left(\zeta_{i}^{*}(\cdot)\right)_{i \in I}$, we can then derive an ex-ante equilibrium distribution over states $s=(y, \zeta)$ as

$$
\mathrm{P}(s=(y, \zeta))=\mathrm{P}(y) \sum_{z \in Z: \zeta_{i}^{*}\left(z_{i}\right)=\zeta_{i} \forall i \in I} \mathrm{P}(z \mid y),
$$

using (6.1). This would be the measure used by the Social Planner when measuring financial centrality, since she has to integrate over agents' signals from an ex ante perspective, according to the assumed common prior distribution $\beta_{0}$.

\subsection{A General Derivation of financial centrality with Endogenous Market Partici-}

pation. In this section we formally define, in this setting, what we mean by endogenous market participation environments. Intuitively, an environment has endogenous market participation if the income draw is an exogenous variable, and then the market participation distribution is then determined by this draw. Formally, is an environment with the following timeline: (1) income distribution is drawn $y \sim F(y)$ (as seen before), and then (2) market participation is drawn according to $\zeta \sim \mathrm{P}(\zeta \mid y)$.

Of course, this type of environment generally has the property that $\zeta \not \perp y$. Of course, any joint distribution for $s=(y, \zeta)$ can be decomposed in this manner: what makes an environment have endogenous market participation is that $y$ is exogenous to market participation $\zeta$. Keeping

${ }^{5}$ We focus on pure strategies for simplicity of exposition. 
the example in mind, recall that in general, without endogenous participation, our notion of financial centrality thus far has been

$$
F C_{i}=\frac{\partial \mathcal{L}}{\partial y_{i}}=\mathbb{E}_{s}\left[\zeta_{i} q(s)\right]
$$

where we used the fact that the joint distribution of $s=(y, \zeta)$ did not change when an agent received an increase in her endowment. However, when market participation is a choice that can depend on endowments, then there might be precisely such an effect.

We can show that in this case that there is a natural extension, with a second term reflecting the effect that changing the endowment of an agent has on market participation.

Proposition 6.1. Suppose the environment has endogenous market participation, and that $c(\cdot)$ solves 2.1. Then financial centrality as defined in 3.1 can be written as:

$$
F C_{i}:=\mathbb{E}_{s}\left[\zeta_{i} q(s)\right]+\mathbb{E}_{s}\left\{\left[\sum_{j \in I} \lambda_{j} u_{j}\left(c_{j}(s)\right)\right] \mathbf{S}_{i}(\zeta \mid y)\right\}
$$

where $\mathbf{S}_{i}(\zeta \mid y):=\frac{\partial \log \mathrm{P}(\zeta \mid y)}{\partial y_{i}}$ is the score of the likelihood function $\mathrm{P}(\zeta \mid y)$ with respect to $y_{i}$.

The first term is as before, but the second term looks at a Pareto and utility weighted change in the equilibrium probability of configuration of participation. Basically, by adding $z_{i}$ to $i$, the term looks at the percent change in the probability of each participation configuration weighted by a Pareto-weighted utility term. So holding fixed our notion of centrality without taking the endogenous participation's effect on the configuration likelihoods, this notion of centrality makes an agent more central if the liquidity injection corresponds to an increase in participation configurations where more valuable agents enter.

Proof. For simplicity of exposition, assume a finite state space (i.e., $y$ is a discrete random variable), so the Lagrangian is

$$
\mathcal{L}=\sum_{y \in Y} \sum_{\zeta \in\{0,1\}^{n}}\left[\sum_{j \in I} \lambda_{j} u_{j}\left(c_{j}\right)+\hat{q}(y, \zeta) \sum_{j \in I} \zeta_{j}\left(y_{j}-c_{j}\right)\right] \mathrm{P}(\zeta \mid y) \mathrm{P}(y) .
$$

Using the envelope theorem, we get that

$$
\begin{aligned}
F C_{i} & =\frac{\partial \mathcal{L}}{\partial y_{i}} \\
& =\sum_{y \in Y} \sum_{\zeta \in\{0,1\}^{n}} \zeta_{i} \hat{q}(y, \zeta) \mathrm{P}(\zeta \mid y) \mathrm{P}(y) \\
& +\sum_{y \in Y} \sum_{\zeta \in\{0,1\}^{n}}\left[\sum_{j \in I} \lambda_{j} u_{j}\left(c_{j}\right)+\hat{q}(y, \zeta) \sum_{j \in I} \zeta_{j}\left(y_{j}-c_{j}\right)\right] \frac{\partial \mathrm{P}\left(\zeta \mid y_{i}\right)}{\partial y_{i}} \mathrm{P}(y)
\end{aligned}
$$

and using the facts that $q(y, \zeta)=\hat{q}(y, \zeta) / \mathrm{P}(y, \zeta)$ and complementary slackness implies

$$
\hat{q}(y, \zeta) \sum_{i \in V} \zeta_{i}\left(y_{i}-c_{i}\right)=0
$$


for all $(y, \zeta)$. We can simplify this expression as

$$
F C_{i}=\mathbb{E}_{y, \zeta}\left\{\zeta_{i} q(y, \zeta)\right\}+\mathbb{E}_{y, \zeta}\{\sum_{j \in I} \lambda_{j} u_{j}\left(c_{j}\right) \underbrace{\frac{\partial \mathrm{P}\left(\zeta \mid y_{i}\right)}{\partial y_{i}} \frac{1}{\mathrm{P}\left(\zeta \mid y_{i}\right)}}_{:=\mathbf{S}_{i}\left(\zeta \mid y_{i}\right)}\}
$$

proving the desired result.

In the model proposed in subsection 6.1, we assume that the credit line policy $t=\left(t_{j}\right)_{j \in J}$ from section 3.1 is common knowledge among agents, and hence the policy has no effect on the information agents have access to. It does, however, affect the relative utility of market access. That is, the market access strategy (given transfer $t_{i} \geq 0$ ) is

$$
\zeta_{i}^{*}\left(\theta_{i} \mid t\right)=1 \Longleftrightarrow \mathbb{E}_{s}\left\{u_{i}\left[c_{i}\left(y_{i}+t_{i}, y_{-i}, \zeta\right)\right]-u_{i}\left(y_{i}\right) \mid \theta_{i}\right\} \geq \kappa_{i} .
$$

If $c_{i}(\cdot)$ is a weakly increasing in own endowment (e.g.: $c_{i}(s)=\bar{y}_{\zeta}$ in an environment with an utilitarian planner, and agents with homogeneous preferences) the transfer $t_{i}$ acts as a subsidy for market participation, increasing the set of signals $\theta_{i}$ for which condition 6.2 is satisfied. However, since the transfer policy is assumed to be common knowledge, this also affects the marker participation decisions of other agents. If $c_{i}$ is weakly increasing for all agents (e.g. also $\left.c_{i}(s)=\bar{y}_{\zeta}\right)$ then other agents also have higher incentives to access the market, since it is more likely that $i$ will be trading, and $i$ is more valuable, since $i$ increases aggregate income whenever she trades. We summarize this result in the following corollary.

Corollary 6.1. Take the model of Private Participation and Costly Market Access of Section 6.1, and $\lambda \in \Delta^{n}$. If the allocation $c(\cdot)$ solving 2.1 is non-decreasing in $y$, then $F C_{i}>$ $\mathbb{E}_{s}\left[\zeta_{i} q(s)\right]$.

6.3. Team Production Environments. Another type of endogenous market participation considers a setting where market participation shocks are determined exogenously first and then the income distributions for agents with market access depend on the identities of those trading. Formally, the timing on the resolution of uncertainty would be as follows: (1) Market participation $\zeta$ is drawn according a distribution $G(\zeta) ;(2)$ Income distribution is drawn from $y \sim F(y \mid \zeta)$. A leading example of such an environment is one of team production. Agents without market access draw income from their autarky income distribution $y_{i} \sim F_{i}\left(y_{i}\right)$. However, once agents are drawn together to form a market, income is drawn jointly, and then agents can divide aggregate income draws amongst them in any feasible consumption allocation.

Models like this also show correlation between market participation and income. However, it is straightforward to see that in such models, financial centrality is simply $F C_{i}=\mathbb{E}_{s}\left\{\zeta_{i} q(s)\right\}$ as before. This is simply because the injection policy of giving an injection to agent $i$ has no effect on the market participation distribution, since it is assumed here to be exogenous to income draws. But unlike the baseline model, income and market participation are now not 
independent, and the expectation has to be calculated over market participation and income shocks jointly.

\section{Market Formation Process}

Throughout the paper we have characterized financial centrality abstractly without modeling explicitly the market formation process. In this section we briefly provide a few examples of market making or matching models which are admittedly stylized, but intended to be pedagogical and in certain contexts applicable.

In the homogeneous case, since

$$
F C_{i} \propto \mathbb{E}_{\zeta}\left\{\zeta_{i}\left(1+\frac{\gamma}{n(\zeta)}\right)\right\}
$$

we need to calculate $\mathbb{E}\left\{\frac{1}{n(\zeta)} \mid \zeta_{i}=1\right\}$ and $\mathrm{P}\left(\zeta_{i}=1\right)$. Here we propose some examples of these market making processes.

7.1. Warm-up: Degree Model. The first example imagines a network where in every period, a single node is chosen as a host and all of its neighbors are activated to trade.

Let $g=(I, E)$ denote the network with $E$ the set of edges and $g_{i j}=1\{i j \in E\}$. For simplicity this is an undirected, unweighted graph and assume each node has a self-loop $\left(g_{i i}=\right.$ 1). Let $d_{i}:=\sum_{j} g_{i j}$ denote the degree of node $i$ and let $N_{i}:=\left\{j \in I: g_{i j}=1\right\}$ denote the neighborhood of $i$.

Market participation is drawn as follows. With probability $z_{i}=\frac{1}{n}$, each agent is selected to be the host. Then $\zeta_{i}=1$ and also $\zeta_{j}=1\left\{j \in N_{i}\right\}$. We can compute financial centrality as

$$
F C_{i}=\frac{1}{n}\left\{d_{i}+\gamma \sum_{j} \frac{g_{i j}}{d_{j}}\right\} .
$$

Agents who have larger neighborhoods are more central (from the $d_{i}$ term), but in particular agents that have neighbors who have smaller neighborhoods are more central (from the $\frac{1}{d_{j}}$ term). The notion of centrality derived from this model may be quite different from traditional notions of centrality, such as betweenness, Bonachich, or eigenvector centrality, among others. In Figures 7.1a and 7.1b, we compare two agents $i$ and $j$ in different parts of a large network (so $n$ is the same for both of them). Observe that agent $j$ in Figure 7.1b is more central than the one in Figure 7.1b, $i$, according to most commonly used centrality measures, since she can reach more agents in the same number of steps (higher eigenvector centrality, for example). However, the agent $j$ is less financially central than $i$, since (a) it has the same probability of having market access, but (b) the markets she has access to are bigger (in the first order stochastic dominance sense) to those that agent $i$ reaches, and is hence less important. This is because of the logic of consumption variance reduction: a dollar given to the agent $i$ will reduce consumption variance a lot more than agent $j$.

7.2. General Poisson Models. The second example generalizes the above. Let $z_{i} \in[0,1]$ denote the probability that an agent gets selected as the host. Then let $\mathbf{p}$ denote a matrix 


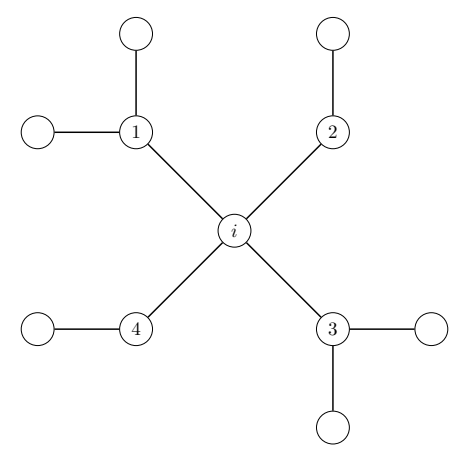

(A)

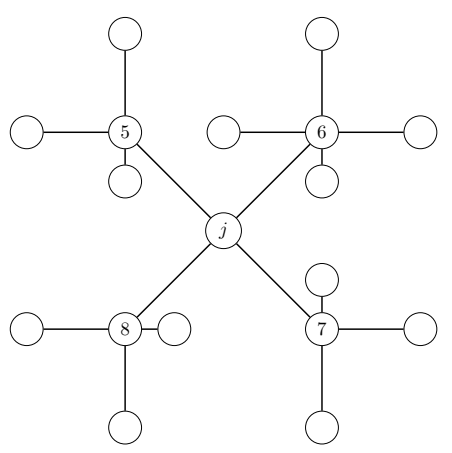

(B)

Figure 7.1. Two agents in a large network, $i$ and $j$, in Panels (A) and (B) respectively. Standard measures of centrality would typically treat $j$ as being more central than $i$, ceteris paribus, since they have the same degree and $j$ 's neighbors' degrees are higher. Our notion of financial centrality ranks $F C_{j}<$ $F C_{i}$.

with entries $\mathbf{p}_{i, j}$ denoting the probability that $j$ is in the market when $i$ is the host, which is independent across $j$. We set $\mathbf{p}_{i, i}=1$.

This nests some obvious special cases. For example, in the degree model above, $\mathbf{p}=g$. Another example is an invitations model, where each host sends out an invitation to all the other network members which arrives with probability that declines in the network distance $\delta_{i j}$ between $i$ and $j$. Then $\mathbf{p}_{i, j}=\alpha^{\delta_{i j}}$ for some probability $\alpha$ (Jackson and Wolinsky, 1996).

It is useful to define an individual specific parameter, which is the expected number of individuals in the trading room when $i$ is selected as host, $\nu_{i}$. This can be computed as $\nu_{i}:=\sum_{j} \mathbf{p}_{i j}$. To characterize financial centrality, we need to know the expected sizes of the trade rooms when $i$ is host and conditional on $i$ being in the room, integrating across the other possible hosts. Two auxiliary random variables will be very useful in the rest of the section: $\mathbf{n}_{-i}=\left(n_{\zeta}-1\right) \mid i$ is host, and $\mathbf{n}_{-j, i}=\left(n_{\zeta}-2\right) \mid j$ hosts $\& \zeta_{i}=1$. This means that whenever $i$ hosts, market size is $n_{\zeta}=1+\mathbf{n}_{-i}$, and whenever $j$ hosts, and we condition on $i$ accessing the market, then market size is $n_{\zeta}=2+\mathbf{n}_{-j, i}$. This auxiliary random variables have range from 0 to $k \in\{n-1, n-2\}$, and based on our assumptions, we have

$$
\mathbf{n}_{-i} \sim \sum_{k \neq i} \operatorname{Bernoulli}\left(\mathbf{p}_{i, k}\right) \text { and } \mathbf{n}_{-j i} \sim \sum_{k \notin\{j, i\}} \operatorname{Bernoulli}\left(\mathbf{p}_{j, k}\right),
$$

where these Bernoulli distributions are independent, with success probabilities strictly less than $1 .{ }^{6}$ These distributions are also called Poisson Binomial distributions, and have been extensively studied in the literature. We will write $X \sim P B(\mathbf{p})$ with $p=\left(p_{1}, p_{2}, \ldots, p_{k}\right)$ the vector of success probabilities of each Bernoulli trial. This distribution, in some cases, can be

\footnotetext{
${ }^{6}$ Exact and approximation methods for calculating expectations of market sizes are sensitive to the assumption of interior (i.e., in $(0,1))$ success probabilities. This is the reason for the need to define the random variables $\mathbf{n}_{-i}$ and $\mathbf{n}_{-j i}$.
} 
well approximated by a Poisson distribution. ${ }^{7}$ In this model, we have that $\mathbf{n}_{-i} \sim P B\left(\mathbf{P}_{i}\right)$ and $\mathbf{n}_{-j i} \sim P B\left(\mathbf{P}_{-j, i}\right)$ where $\mathbf{P}_{i}=\left(\mathbf{p}_{i, k}\right)_{k \neq i} \in[0,1]^{n-1}$ and $\mathbf{P}_{-j, i}=\left(\mathbf{p}_{j, k}\right)_{k \notin\{i, j\}} \in[0,1]^{n-2}$. These random variables are useful to write our approximation to financial centrality as

$$
\begin{aligned}
F C_{i} \approx \hat{F C_{i}} & :=\mathrm{P}\left(\zeta_{i}=1\right) \times\left[1+\gamma \mathbb{E}\left(\frac{1}{n_{\zeta}} \mid \zeta\right)\right] \\
& =p_{i}\left[1+\gamma z_{i} \mathbb{E}\left(\frac{1}{1+\mathbf{n}_{-i}}\right)+\gamma \sum_{j \neq i} z_{j} \mathbb{E}\left(\frac{1}{2+\mathbf{n}_{-j i}}\right)\right],
\end{aligned}
$$

where $p_{i}=\mathrm{P}\left(\zeta_{i}=1\right)$. Therefore, we need to calculate the inverse moments $\mathbb{E}(1 / 1+X)$ and $\mathbb{E}(1 / 2+X)$ for $n$ Poisson Binomial random variables; $X=\mathbf{n}_{-i}$ and $X=\mathbf{n}_{-j i}$ for all $j \neq i$. Hong (2013) provides a general survey on the commonly used methods to calculate explicitly the probability function of Poisson Binomial distributions, using either recursive or Discrete Fourier Transform methods, which are fairly fast even with large $n .{ }^{8}$ We also survey results (starting with Le Cam (1960)) that show that if the expected number of successes of a Poisson Binomial distribution is sufficiently low (corresponding in this case with low expected market sizes), then it can be well approximated by a Poisson distribution. ${ }^{9}$ In the context of this model, it means that if $\mathbb{E}\left(\mathbf{n}_{-i}\right)=\nu_{i}-1$ is small (relative to $n$ ), then we can approximate $\mathbf{n}_{-i} \sim$ Poisson $\left(\nu_{i}-1\right.$ ) and $\mathbf{n}_{-j i} \sim \operatorname{Poisson}\left(\nu_{-j i}\right)$, where $\nu_{-j i}=\mathbb{E}\left(n_{-j i}\right)=\sum_{k \notin\{i, j\}} \mathbf{p}_{j k}=\nu_{j}-\mathbf{p}_{j i}-1$.

If $X \sim \operatorname{Poisson}(\nu-1)$, then $\mathbb{E}(1+X)^{-1}=m_{1}(\nu):=[1-\exp (1-\nu)] /(\nu-1)$ and $\mathbb{E}(2+X)^{-1}=$ $m_{2}(\nu):=\left[1-m_{1}(\nu)\right] /(\nu-1)$, both strictly decreasing functions of $\nu \geq 1$. Using these formulas, we can then approximate $\hat{F C}$ by:

$$
\hat{F C_{i}} \approx p_{i} \times\left\{1+\gamma z_{i} m_{1}\left(\nu_{i}\right)+\gamma \sum_{j \neq i} z_{j} m_{2}\left(\nu_{j}-\mathbf{p}_{j, i}\right)\right\} .
$$

This shows the following. First, nodes with a larger expected reach as measured by $\nu_{i}$ are more central (as long as $n$ is large enough relative to $\gamma$ ). Second, nodes that have larger expected inverse room size when they are hosts are more central. Third, $i$ is more central when $\mathbf{p}_{j, i}$ increases, particularly when $\nu_{j}$ is small. So when $j$ tend to invite small rooms as hosts, but $i$ is likely to be in such a $j$ 's room, then $i$ is more valuable.

\footnotetext{
${ }^{7}$ Le Cam (1960) provided bounds on the error of approximation, which were improved by Stein (1986); Chen (1975), and Barbour and Hall (1984); Sason (2013) show that if $\hat{X}$ is the poisson approximation (with mean $\lambda=\sum_{i} p_{i}$, then $d_{T V}(X, \hat{X}) \leq\left(1-e^{-\lambda}\right) \sum_{i} p_{i}^{2} / \lambda$, where $d_{T V}(\cdot)$ denotes the total variation distance. This approximation will then typically be valid when its expected value is not too large.

${ }^{8}$ Chen and Liu (1997) show stable (i.e. non-alternating) methods are $O\left(n^{2}\right)$, which would make the calculation of financial centrality of a given agent be $O\left(n^{3}\right)$. Discrete Fourier Methods are usually much faster (Fernández and Williams (2010)). See Hong (2013) for a general survey on the existing exact and approximating methods. ${ }^{9}$ This is not the only approximation studied in the literature. In models where the expected market size is high, Gaussian approximations behave rather well (see Volkova (1996), Hong (2013)). If success probabilities are similar (i.e. the variance $\sigma_{p}^{2}:=n^{-1} \sum_{i}\left(p_{i}-\bar{p}\right)^{2}$ is small enough) then approximation to a Binomial distribution is fairly accurate (Ehm (1991); Barbour et al. (1992))
} 
A special case are symmetric models, where $z_{i}=1 / n$ for all $i$ and $\mathbf{p}_{i, j}=\mathbf{p}_{j, i}$ (e.g. the model $\mathbf{p}_{i, j}=\alpha^{\delta(i, j)}$, since distance is symmetric). In this case

$$
F C_{i}=\frac{1}{n} \nu_{i}\left\{1+\gamma \times \frac{1}{n} m_{1}\left(\nu_{i}\right)+\gamma \sum_{j \neq i} \frac{1}{n} m_{2}\left(\nu_{j}-\mathbf{p}_{j, i}\right)\right\} .
$$

This has the advantage that the centrality of agent $i$ depends solely on the expected market size of each agent (as a host) that she gets connected to, and the probability that she connects to them. The marginal value of the inverse room size effect when $i$ is the host, proportional to $\nu_{i} \times m_{1}\left(\nu_{i}\right)$, declines in $\nu_{i}$ if and only if $\nu_{i} \geq 2.79$ (there is a positive effect in $\mathrm{P}\left(\zeta_{i}=1\right)$, but an offsetting negative effect in $m_{1}\left(\nu_{i}\right)$ )

If we want to calculate centrality exactly, we can still use the calculation of the exact pdf of $\mathbf{n}_{-i}$ and $\mathbf{n}_{-j i}$ to get the exact Financial Centrality. For example, in the CARA+normal model with homogeneous preferences and independent and identically distributed income draws, we know that $F C_{i}=\mathbb{E}_{\zeta}\left\{\zeta_{i} \exp \left(\gamma / n_{\zeta}\right)\right\}$, which can be decomposed as

$$
F C_{i}=p_{i}\left\{z_{i} \mathbb{E}\left[\exp \left(\frac{\gamma}{1+\mathbf{n}_{-i}}\right)\right]+\sum_{j \neq i} z_{j} \mathbb{E}\left[\exp \left(\frac{\gamma}{2+\mathbf{n}_{-j i}}\right)\right]\right\}
$$

and then be calculated explicitly using the distributions for $\mathbf{n}_{-i}$ and $\mathbf{n}_{-j i}$.

7.3. Transaction Chains. In the Poisson models, for $j \neq k \neq i$, note that $\zeta_{j} \perp \zeta_{k}$ conditional on $i$ hosting. But trading groups may be determined dynamically, along a chain of meetings. In this case the study of random walks on graphs provides the right vocabulary to capture this.

We can model this in a simple way, though the analytic characterization is hard to come by. Let $\left(z_{i}\right)_{i \in[n]}$ denote the probabilities that each node is the host, let $\left(p_{i j}\right)_{i, j \in[n]}$ denote the probability that $i$ meets $j$, and let $\beta$ be the probability that at each stage the chain continues. With complementary probability $1-\beta$, the chain terminates exogenously. However, the chain also terminates if an agent is revisited (and hence no new agents are added to the market).

This process, at termination, determines the size of the trading room. While it is easy to describe, and easy to simulate, it is hard to analytically compute moments for the distribution of $\frac{1}{n_{\zeta}}$ (Aldous and Fill, 2002; Durrett, 2007), even if chains are not terminated upon revisiting an agent. This is because what matters is the number of distinct agents in the market, not just the number of steps the chain makes (which, in that case, would simply follow a Geometric random variable).In the special case with large $n, z_{i}=1 / n, \beta=1$ (no random exogenous termination) and $p_{i j}=1 / d_{i}$ (i.e. uniform random walk, with equal probability among first degree neighbors) and $g$ comes from an Erdös-Renyi process, Tishby et al. (2017) get closed form expressions for the distribution of chain length (or market size in our setup), showing that it follows a product of an exponential and a Rayleigh distribution ${ }^{10}$

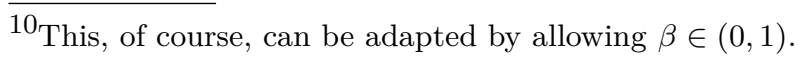




\section{8. (Constrained) Efficient Solution Concepts Determining Pareto Weights}

The analysis so far has been normative: that is, how a planner with given preferences should measure the relative importance (in terms of marginal increase in welfare) of increasing the endowment of agents whenever they are able to trade. In this section, we propose an alternative, positive approach. We want to investigate whether in some feasible solution concepts, agents with higher centrality get rewarded by higher consumption, which in the CARA model with normally distributed income, translates into a higher Pareto weight in the planner's representation of the consumption allocation. We focus on this environment throughout this section.

We first show that if the solution concept is cooperative Nash bargaining, then there is a positive linear relationship between the representing Pareto weight of an agent and her financial centrality measure. However, as we saw in Section 5, financial centrality is itself a function of the Pareto weights vector, which makes the determination of the representing Pareto weights vector a fixed point equation problem. Secondly, we study the Kalai-Smorodinsky bargaining solution, which unlike the Nash bargaining solution, does not explicitly depend on the financial centrality of the agents. However, the representing Pareto weights (which, again, map into higher consumption for the agent in the CARA preferences environment) does depend on moments that typically make financial centrality of an agent higher, such as smaller market sizes conditional on the agent being trading.

8.1. Nash Bargaining. Suppose agents decide the social contract by bargaining among themselves. Agents receive an expected utility $U_{i}=\mathbb{E}\left[u_{i}\left(c_{i}(s)\right)\right]$ in a contract. If they reject the proposed social contract, then agents get their "disagreement point", or autarky value, $U_{i}^{\text {aut }}=\mathbb{E}_{y_{i}}\left[u_{i}\left(y_{i}\right)\right]$. The social contract is the choice of a feasible consumption allocation $c(s)=\left\{c_{i}(s)\right\}_{s=(y, \zeta)}$. If the bargaining process satisfies Pareto optimality, linearity in utilities and independence of irrelevant alternatives, then the optimal contract solves

$$
\max _{c(s)} \prod_{i \in I}\left\{\mathbb{E}_{s}\left\{\zeta_{i} u_{i}[c(s)]+\left(1-\zeta_{i}\right) u_{i}\left(y_{i}\right)\right\}-\mathbb{E}_{y_{i}}\left[u_{i}\left(y_{i}\right)\right]\right\}^{\alpha_{i}}
$$

subject to $\sum_{i} \zeta_{i} c_{i}(s) \leq \sum_{i} \zeta_{i} y_{i}$, for some vector $\alpha$ such that $\sum_{i} \alpha_{i}=1$ and $\alpha_{i} \geq 0$. This is equivalent to solve the following program

$$
\begin{aligned}
& \max _{c(s)} \sum_{i \in I} \alpha_{i} \ln \left\{\mathbb{E}_{s} \zeta_{i}\left[u_{i}\left(c_{i}(s)\right)-u_{i}\left(y_{i}\right)\right]\right\} \\
& \text { s.t }: \sum_{i \in I} \zeta_{i} c_{i}(s) \leq \sum_{i \in I} \zeta_{i} y_{i} \text { for all }(y, \zeta) .
\end{aligned}
$$

The first order conditions of this problem are

$$
\frac{\alpha_{i}}{\mathbb{E}_{s} \zeta_{i}\left[u_{i}\left(c_{i}(s)\right)-u_{i}\left(y_{i}\right)\right]} u^{\prime}\left(c_{i}(s)\right)=q(s)
$$

where, again, $q(s)$ are the Lagrange multipliers of the resource constraints. Therefore, the equivalent Pareto weight in the Planner's problem is exactly 


$$
\lambda_{i}=\frac{\alpha_{i}}{\mathbb{E}_{s} \zeta_{i}\left[u_{i}\left(c_{i}(s)\right)-u_{i}\left(y_{i}\right)\right]}
$$

In the next proposition, we investigate the fix point equation for the CARA normal model.

Proposition 8.1. Suppose $u_{i}(c)=-r_{i}^{-1} \exp \left(-r_{i} c\right)$ and $y \sim \mathcal{N}(\mu, \Sigma)$. Then, the Pareto weights associated with the Nash bargaining solution with bargaining weights $\alpha \in \Delta^{n}$ satisfy the following fix point equations:

$$
\lambda_{i}=\frac{\alpha_{i} r_{i}+F C_{i}(\lambda)}{p_{i} \exp \left(-r_{i} \mu_{i}+\frac{r_{i}^{2}}{2} \sigma_{i}^{2}\right)} \text { for all } i .
$$

Proof. See Appendix A.

This proposition shows why the Nash Bargaining Solution is a nice application in our setting. In the fixed point equation, holding everything else fixed, agents with higher financial centrality have also higher Pareto weights (which, of course, is the result of a fixed point equation). This suggests that, if agents bargain over risk sharing contracts, holding autarky as a threat point of the negotiation, then agents with higher centrality should have higher portions of aggregate income.

In particular, for the symmetric Nash bargaining solution $\left(\alpha_{i}=1\right)$ and homogeneous preferences and i.i.d income, we get that the representing Pareto weights are not uniform $\left(\lambda_{i} \neq 1 / n\right)$ but rather satisfy

$$
\ln \left(\lambda_{i}\right)=\kappa+\ln \left[r+F C_{i}(\lambda)\right]-\ln \left(p_{i}\right)
$$

so the heterogeneity in the market participation process has a bite, unlike (as we saw above) the Walrasian equilibrium with the same preferences.

8.2. Kalai-Smorodinsky Bargaining. The second most used bargaining solution in the literature is the Kalai-Smorodinsky solution. It also gives closed form solutions to Pareto weights, and are expressed as a function of fundamentals of the environment, rather than a fixed point equation.

The most important parameter in the bliss point. The bliss point for agent $i, \bar{U}_{i}$ is defined as the utility she would achieve if she consumed all the available income in the market in every state where she can trade, and only her own income otherwise:

$$
\bar{U}_{i}:=\mathbb{E}_{\zeta, y}\left[\zeta_{i} u_{i}\left(\sum_{j} \zeta_{j} y_{j}\right)+\left(1-\zeta_{i}\right) u_{i}\left(y_{i}\right)\right]
$$

and $\bar{U}=\left(\bar{U}_{1}, \bar{U}_{2}, \ldots, \bar{U}_{n}\right)$. Likewise, the disagreement point $\underline{U}_{i}$ is the value of autarky in this environment for each agent

$$
\underline{U}_{i}:=\mathbb{E}_{y_{i}}\left[u_{i}\left(y_{i}\right)\right]
$$

and $\underline{U}:=\left(\underline{U}_{1}, \underline{U}_{2}, \ldots, \underline{U}_{n}\right)$. The Kalai-Smorodinsky solution consists on finding the linear combination of $\bar{U}$ and the $\underline{U}$ that lies on the Pareto frontier of the utility possibility set; i.e, 
find $\alpha \in[0,1]$ such that $\alpha \bar{U}+(1-\alpha) \underline{U} \in \mathbb{P}(\mathcal{U})$, and the solution is $U^{*}=\alpha \bar{U}+(1-\alpha) \underline{U}$. Since $\bar{U}>\underline{U}$, the Kalai-Smorodinsky solution here would be

$$
\max _{\alpha \in[0,1],\left\{c_{i}(y, \zeta)\right\}_{i \in I}} \alpha
$$

subject to

$$
\begin{cases}\mathbb{E}_{y, \zeta}\left[\zeta_{i} u_{i}\left(c_{i}(y, \zeta)\right)+\left(1-\zeta_{i}\right) u_{i}\left(y_{i}\right)\right] \geq \alpha \bar{U}_{i}+(1-\alpha) \underline{U}_{i} & \text { for all } i \\ \sum \zeta_{i} c_{i}(y, \zeta) \leq \sum \zeta_{i} y_{i} & \text { for all }(\zeta, y)\end{cases}
$$

One of the most attractive properties of the Kalai-Smorodinsky solution is that the Pareto weights derived from it have a closed form formula, and is not a fixed point equation (as in the Nash Bargaining solution case).

Proposition 8.2. If the risk sharing contract is the Kalai-Smorodinsky solution over the utility possibility set, then the Pareto weights associated with the solution are

$$
\lambda_{i}=\frac{1}{\mathbb{E}_{s}\left\{\zeta_{i}\left[u_{i}(Y(s))-u_{i}\left(y_{i}\right)\right]\right\}}
$$

where $Y(s)=\sum_{j} \zeta_{j} y_{j}$ is the aggregate income in state $s=(y, \zeta)$. If $u_{i}(c)=-r_{i}^{-1} \exp \left(-r_{i} c\right)$ and $y \sim \mathcal{N}(\mu, \Sigma)$, then

$$
\lambda_{i}=\frac{\beta}{p_{i} \times \mathbb{E}_{\zeta}\left\{\exp \left(-r \mu+\frac{\sigma^{2}}{2}\right)-\exp \left[n_{\zeta}\left(-r \mu+\frac{\sigma^{2}}{2}\right)\right] \mid \zeta_{i}=1\right\}}
$$

where $\beta=r /\left(-r \mu+\sigma^{2} / 2\right)$.

Proof. See Appendix A.

Corollary 8.1. In the CARA normal model, with homogeneous preferences and i.i.d income shocks, if $\mu>\frac{r}{2} \sigma^{2}$, then Lagrange multiplier are decreasing (in the FOSD sense) in market size.

Unlike Nash Bargaining, Pareto weights in this environment have a closed form solution, so comparative statics are easier to interpret, and the comparative statics are the same as the one suggested by the Nash Bargaining fixed point equations.

\section{An Empirical Example}

We now take our perspective to the data. The goal of this section is to demonstrate, with an observational analysis, that there is empirical content in our theoretical approach. We make no causal claims here. We look at the Townsend Thai village data over 15 years, and we focus on 338 households across 16 villages where we have detailed data on consumption, income, and transactions across villagers (Samphantharak and Townsend, 2010). In particular, in this setting we have variation in the number of transactions per time period. We use whether a household has reported making or receiving a transfer to any other household in the given month as a measure of being active in the network in a given period. 
Our theory suggests that nodes that provide more value, those with higher measures of financial centrality, are exactly those that are in the market when the market is thin (in a generalized sense including few active traders and greater per-trader-volatility in income).

We proceed in two steps. First, we need a measure that reflects $F C_{i}=\frac{\partial V}{\partial t_{i}}$. As we show in subsections 8.1 and 8.2, if Pareto weights are determined by Nash bargaining or KalaiSmorodinsky bargaining, then a more financially central individual $i$ has a higher Pareto weight $\lambda_{i}$. So, though we do not observe financial centrality, we can use observations on consumption in panel data to obtain an estimate of a function for each agent $i$ which is monotonically increasing in the Pareto weight $\lambda_{i}$. Specifically we obtain the household fixed-effect of consumption, using only active periods:

$$
c_{i v t}=\alpha_{i}+\beta y_{i v t}+\delta_{t v}+\epsilon_{i v t}
$$

where $t$ is indexing a set of active periods, $\alpha_{i}$ is a household fixed-effect, and $\delta_{v t}$ is a villageby-time fixed effect. Under CARA utility the $\alpha_{i}$ is a monotone function of Pareto weights $\lambda_{i}$

We also know from our theory the crucial components in our financial centrality measure, market thinness. So we next compute a measures of market thinness for each household by factor,

$$
\rho_{i}^{\zeta}:=\operatorname{cov}_{t}\left(\zeta_{i t}, \frac{1}{n_{v t}}\right) \text { and } \rho_{i}^{\sigma}:=\operatorname{cov}_{t}\left(\zeta_{i t}, \bar{\sigma}_{t}\right),
$$

where $n_{v t}$ is the number of active participants in period $t$ in a village $\mathrm{v}$, computed from the transfers data as mentioned, and where $\bar{\sigma}_{t}^{2}:=\frac{1}{n_{\zeta_{t}}} \sum_{i, j} \zeta_{i t} \zeta_{j t} \widehat{\sigma}_{i, j, t}$ is an estimate of the volatility at period $t$, where $\hat{\sigma}_{i, j, t}$ is the measured covariance between households $i$ and $j$ 's income.

Finally we check on the relationship of these two pieces, implied Pareto weights and crucial components of financial centrality, by running a multi-variate regression

$$
\alpha_{i}=\beta_{0}+\beta_{1} \rho_{i}^{\zeta}+\beta_{2} \rho_{i}^{\sigma}+X_{i}^{\prime} \beta_{3}+u_{i}
$$

where $X_{i}$ is a polynomial of wealth. Our theory suggests that $\beta_{1}>0$ and $\beta_{2}>0$. We note that this is an observational claim, but it is not mechanical: that those who are present exactly when the market is thin tend to receive a greater mean consumption, even conditional on wealth, is consistent with our model.

Table 1 presents the results. Columns 1-2 and 4-5 include each measure of market thinness when the agent enters one-by-one and columns 3 and 6 include them together. Columns 13 include no subsequent controls, whereas columns 4-6 include a third-degree polynomial in wealth. We see a one-standard deviation increase in the tendency to enter when the market is thin in numbers is associated with a corresponding 0.095 standard deviation increase in mean consumption (column 1). Similarly, a one-standard deviation increase in the tendency to enter when the market is thin in the sense of high volatility is associated with a corresponding 0.103 standard deviation increase in the mean consumption (column 2). These estimates are stable to being jointly included (column 3), as well as when we include wealth controls. 
TABle 1. Do Pareto weights correlate with measures of market thinness when the agent is active?

\begin{tabular}{lcccccc}
\hline & $(1)$ & $(2)$ & $(3)$ & $(4)$ & $(5)$ & $(6)$ \\
& $\alpha_{i}$ & $\alpha_{i}$ & $\alpha_{i}$ & $\alpha_{i}$ & $\alpha_{i}$ & $\alpha_{i}$ \\
\hline$\rho_{i}^{\xi}$ & & & & & & \\
& $0.095^{* *}$ & & $0.112^{* *}$ & $0.078^{*}$ & & $0.093^{* *}$ \\
$\rho_{i}^{\sigma}$ & $(0.041)$ & & $(0.045)$ & $(0.041)$ & & $(0.044)$ \\
& & $0.103^{* *}$ & $0.118^{* *}$ & & $0.121^{* *}$ & $0.131^{* *}$ \\
& & $(0.050)$ & $(0.051)$ & & $(0.051)$ & $(0.052)$ \\
Observation & 338 & 338 & 338 & 338 & 338 & 338 \\
R-squared & 0.016 & 0.018 & 0.040 & 0.060 & 0.074 & 0.088 \\
Controls & None & None & None & Wealth & Wealth & Wealth \\
\hline
\end{tabular}

Notes: Robust standard errors in parentheses. The dependent variable is a (mean zero, standardized) Pareto weight estimate of a given household, obtained from using the vectors of household fixed effects from a regression of consumption on household income. Regressors are each standardized as well. Wealth controls includes a thirddegree polynomial in household wealth. ${ }^{* * *} p<0.01,{ }^{* *} p<0.05, * p<0.1$

Taken together, the results are consistent with a story where agents have determined Pareto weights through a bargaining process, and those who have higher weights and therefore higher financial centrality are precisely those who tend to be active traders when the market is thin either in terms of numbers of individuals or volatility. Our results are, of course, observational and merely suggestive.

\section{Extensions}

In this section we study two extensions that depart from the class of environments we study. One of the most seemingly important restrictions on the models studied so far is the existence of centralized markets: agents either are in autarky or have market access and can trade with any other agent that also has market access. While the bilateral trading chains introduced in subsection 3.3 relaxes this interpretation, it maintains the possibility that any agent is reachable to any other, through a finite sequence of trades, as long as both have market access. In this Section 10.1 we introduce a generalization of the basic environment, allowing for the existence of several segmented markets working in parallel, where agents can only trade among a subset of all agents who have market access. We show that the basic definitions and formulas of financial centrality still hold, if we reinterpret having "market access" to be present in the market where the agent being injected with liquidity is trading at.

Another important assumption, maintained throughout this paper, is that the Social Planner evaluating the marginal value of injected liquidity also is able to implement the allocation $c(\cdot)$ that maximizes her expected utility. However, a relevant case is one where the planner can only influence the economy by the liquidity injection policies introduced in proposed in Section 3.1, and cannot directly choose the allocation herself. This would be the case when the allocation is chosen according to some other solution concept, like Walrasian Equilibrium, multi-player 
bargaining games, and so on. In such situations, the social planner would have to take the consumption allocation as given when measuring the marginal effects of injecting liquidity in this economy. In Section 10.2 we study financial centrality measures under the assumption that the consumption allocation is Pareto optimal, which implies that there exist some representing social preferences (i.e., Pareto weights) for which it would be optimal. We then obtain similar expressions for financial centrality, which now incorporates a term relating the Pareto weights of the Social Planner with the representing Pareto weights of the allocation.

10.1. Segmented Markets . We consider an environment with the same income shocks and preferences, but one where agents may gain access to random, segmented markets. Formally, a market segmentation is a partition $\pi=\left\{m_{1}, m_{2}, \ldots m_{r}\right\}$ over the set of agents $I$; i.e., $\cup_{m \in \pi} m=$ $I$ and $m \cap m^{\prime}=\emptyset$ for all $m \neq m^{\prime}$. In this alternative environment, the relevant state of nature is now $s=(y, \pi)$, where $\pi$ is the market segmentation state, with probability distribution $\mathrm{P}(s)$. We refer to each $m \in \pi$ as a market at state $s$. Let $\mathcal{P}$ be the set of all partitions of $I$ that have positive probability under $\mathrm{P}(s)$. We denote $m(i, \pi) \in \pi$ to be the market (at segmentation $\pi$ ) where $i$ is able to trade. If $m(i, \pi)=\{i\}$ we say $i$ is in autarky at $\pi$, and otherwise we say $i$ has market access at $\pi$. Segmented markets now modify the definition of feasibility of allocations. We say that an allocation $c=\left(c_{i}(s)\right)_{i \in I}$ is feasible if and only if, for all $s=(y, \pi)$ and all $m \in \pi$ we have $\sum_{i \in m} c_{i}(s) \leq \sum_{i \in m} y_{i}$. Clearly, class of environments embedds the Single Market Environments studied before - i.e., markets where any partition $\pi$ in the support is made up of a single multi-agent market $m_{u}(\pi) \subseteq I$ with $\# m_{u}(\pi) \geq 1$, and everyone else being in autarky, and hence we can summarize the state by $s=(y, \zeta)$ where $\zeta_{i}=1 \Longleftrightarrow i \in m_{u}(\pi)$. In general, for a given partition $\pi$ we write $\zeta_{i}^{m} \in\{0,1\}$ for the indicator of whether $i$ has access to market $m$.

Given Pareto weights $\lambda \in \Delta$ and agent $i \in I$, the planner's problem value function of injecting liquidity $t_{i} \geq 0$ to agent $i$ is $V^{s}\left(t_{i}\right):=\max _{\left(c_{j}(y, \pi)\right)_{j \in I}} \mathbb{E}_{s}\left\{\sum_{j \in I} \lambda_{j} u_{j}\left[c_{j}(s)\right]\right\}$ subject to $\sum_{j \in m} c_{j}(s) \leq \sum_{j \in m} y_{j}+t_{i} \zeta_{i}^{m}$ for all $s=(y, \pi)$ and all $m \in \pi$. Financial centrality is now defined in the same as before, for this value function. Intuitively, a planner needs to integrate also over all possible market segmentations in order to assess the marginal value of the liquidity injection policy for agent $i$, since the shadow value of the injection will depend on the market agent $i$ is trading at. We show that the financial centrality measure follows the same formula as in the centralized markets environments, in a "virtual single market economy" where having market access is understood as being able to trade with the agent of interest.

Definition 10.1. Take a segmented market economy $\mathcal{E}$, with distribution over states $\mathbf{P}(y, \pi)$. Define $\mathcal{E}_{i}$ to be a virtual single market economy where all agents have identical preferences over consumption, and the distribution over outcomes $\tilde{\mathbf{P}}(y, \zeta)$ is given by:

$$
\tilde{\mathbf{P}}(y, \zeta)=\mathbf{P}\left((y, \pi) \in Y \times \mathcal{P}:\left\{\begin{array}{l}
(1): j \in m(i, \pi) \text { for all } j: \zeta_{j}=1 \quad \text { and } \\
(2): \# m(i, \pi)>1
\end{array}\right)\right.
$$


i.e., an agent $j \neq i$ has market access on economy $\mathcal{E}_{i}$ only when she is able to trade (i.e., in the same market) with agent $i$ in $\mathcal{E}$.

Proposition 10.1 asserts that financial centrality in a segmented markets economy, follows the same "asset pricing formula" we had in Proposition 3.4, but on the virtual single market economy $\mathcal{E}_{i}$. The proof is quite straightforward, and simply generalizes the proof of Proposition 3.4 , and is therefore omitted.

Proposition 10.1. Suppose $y \perp \pi$. Let $\mathcal{E}$ be a segmented markets economy and $i \in I$. Then, for any $\lambda \in \Delta$, the financial centrality for agent $i$ coincides with the financial centrality of agent $i$ in the virtual single market economy $\mathcal{E}_{i}$. That is,

$$
F C_{i}:=\left.\frac{\partial V^{s}(t)}{\partial t_{i}}\right|_{t=0}=\mathbb{E}_{s=(y, \zeta)}^{\tilde{\mathbf{P}}}\left\{\zeta_{i} q(s)\right\}
$$

where $\mathbb{E}^{\tilde{\mathbf{P}}}(\cdot)$ is the expectation taken w.r.t measure $\tilde{\mathbf{P}}$ defined in 10.1 .

Intuitively, financial centrality only deals with the effect of the increase in agent's $i$ endowment, which can only impact those agents who can trade with her. Because of separability of the planner's preferences over different agents consumptions, the marginal welfare effect on the segmented markets $i$ is trading on have no effect on the welfare evaluation of other segmented markets at the same time. Therefore, whether agents not trading with $i$ are either trading among themselves, or in autarky, is irrelevant when evaluating the policy. Moreover, any two states with generate the same segmented market for agent $i$ are equivalent from the point of view of the planner when evaluating this policy. This result is easily generalized for endogenous market participation economies.

10.2. Passive Planners. In this section, we consider the original environment, but assume the consumption allocation is a primitive of the model (e.g., being determined by a Walrasian Equilibrium). In this setup, the Social Planner can only influence the allocation by making the proposed liquidity injections of Section 3.1. If the social planer has preferences given by $V=\mathbb{E}\left[\sum \lambda_{i} u_{i}\left(c_{i}\right)\right]$, and agents consume according to a (differentiable) allocation $c(\cdot)$, financial centrality would be defined as:

$$
F C_{i}=\mathbb{E}_{s}\left\{\zeta_{i} \sum_{j: \zeta_{j}=1} \lambda_{j} u_{j}^{\prime}\left[c_{j}(s)\right] \frac{\partial c_{j}}{\partial y_{i}}(s)\right\} .
$$

An important case is where $c(\cdot)$ is a (constrained) Pareto optimal allocation; i.e., there exists a representing Pareto weight vector $\varphi$ such that $c(\cdot)$ solves problem 2.1 with $\varphi$ instead of $\lambda$. Also, let $q(s)$ be the usual normalized lagrange multiplier of the resource constraint at state $s$, for this $\varphi$ - planner problem. It is easy to show (see Appendix A) that financial centrality in this setting is

$$
F C_{i}=\mathbb{E}_{s}\left\{\zeta_{i} q(s)\left[\sum_{j: \zeta_{j}=1} \rho_{j} \frac{\partial c_{j}(s)}{\partial y_{i}}\right]\right\},
$$


where $\rho_{j}:=\lambda_{j} / \varphi_{j}^{11}$.

A special case is when the consumption allocation satisfies $\partial c_{j} / \partial y_{i}=n_{\zeta}^{-1}$ whenever $\zeta_{j}=\zeta_{i}=$ 1. This is the case in the CARA model with homogeneous preferences, even if income draws are not Normal (see Appendix A). Whenever this happens, equation 10.2 can be simplified to

$$
F C_{i}=\mathbb{E}_{s}\left\{\zeta_{i} q(s) \times \bar{\rho}_{\zeta}\right\}
$$

where $\bar{\rho}_{\zeta}=n_{\zeta}^{-1} \sum \zeta_{j} \rho_{j}$ is the arithmetic mean of the Pareto weights ratio, and $q(s)$ is the Lagrange multiplier in the Pareto problem with weights $\varphi$. In the CARA-Normal model this then translates into

$$
F C_{i}=\mathbb{E}_{\zeta}\left\{\zeta_{i} \bar{\varphi}_{\zeta} \exp \left(-r \bar{\mu}_{\zeta}\right) \exp \left(\frac{r^{2}}{2} \frac{\bar{\sigma}_{\zeta}^{2}}{n_{\zeta}}\right) \times \bar{\rho}_{\zeta}\right\}
$$

which is the same formula as in Section 5.3, but with an extra term, $\bar{\rho}_{\zeta}:=n_{\zeta}^{-1} \sum \zeta_{j}\left(\lambda_{j} / \varphi_{j}\right)$, the (arithmetic) mean of relative Pareto weights. Another important case where $\partial c_{j} / \partial y_{i}=n_{\zeta}^{-1}$ is an environment where agents are homogeneous preferences and identical and independently distributed random draws. If the allocation comes from a Walrasian equilibrium, we know that the representing Pareto weight is $\varphi_{j}=1$ for all $j$ (see Proposition B.2), and therefore $F C_{i}=\exp (-r \mu) \mathbb{E}_{\zeta}\left\{\zeta_{i} \exp \left(\frac{r^{2}}{2} \frac{\sigma^{2}}{n_{\zeta}}\right) \times \bar{\lambda}_{\zeta}\right\}$, where $\bar{\lambda}_{\zeta}:=n_{\zeta}^{-1} \sum \zeta_{j} \lambda_{i}$ is now the arithmetic mean of the pare-to weight of the social planner. In the baseline case of Section 4 , with homogeneous preferences, i.i.d. income draws and a representing pare-to weight $\varphi_{j}=1$ for all $j$ (so $c_{i}=\bar{y}$ if $\left.\zeta_{i}=1\right)$ we can approximate the centrality measure to $F C_{i} \approx \mathbb{E}_{\zeta}\left\{\zeta_{i}\left(1+\gamma \frac{\sigma^{2}}{n_{\zeta}}\right) \bar{\lambda}_{\zeta}\right\}$, which resembles the centrality measure obtained in Subsection 5.2 for CES and CARA preferences.

\section{Conclusion}

In a number of economic environments, agents in a market share risk, but there is heterogeneity in market access, in the ability to participate in exchange. This is true of financial markets with search frictions and stochastic matching with limited and stochastic market participation financial and monetary models. This is observed in risk-sharing village networks, among other settings. A common, standard model with market participation shocks is used to address the question of how one measures an agent's importance We define the financial centrality of an agent as the marginal social value of injecting an infinitesimal amount of liquidity to that agent.

We show that the most valued agents are not only those who trade often, but are more likely to trade when there are few traders, when income risk is high, when income shocks are positively correlated, when attitudes toward risk are more sensitive in the aggregate, when there are distressed institutions, and when there are tail risks.

We extend our framework to allow for endogenous market participation. Finally, we provide observational evidence from village risk-sharing network data consistent with our model: that

${ }^{11}$ Of course, when $\lambda=\varphi$ we have $\rho_{j}=1$ for all $j$, and since $\sum_{j: \zeta_{j}=1} \partial c_{j}(s) / \partial y_{i}=1$ for all $s: \zeta_{i}=1$, we recover the usual formula in this case. 
the agents that receive the greatest share of the pie are indeed those who are not simply well-connected, but are active precisely when the market is otherwise thin.

Addressing this issue is important for both theory, data, and policy. It allows us to pinpoint both who is providing the greatest marginal value and how one may want to intervene and provide marginal liquidity. .

\section{REFERENCES}

Acemoglu, D., M. A. Dahleh, I. Lobel, and A. Ozdaglar (2010): "Bayesian Learning in Social Networks," .

Acemoglu, D., A. Ozdaglar, and A. Tahbaz-Salehi (2015): "Systemic risk and stability in financial networks," The american economic review, 105, 564-608.

Aldous, D. AND J. Fill (2002): "Reversible Markov chains and random walks on graphs," .

Allen, F. And A. Babus (2009): "Networks in Finance," in Network-based Strategies and Competencies, ed. by P. Kleindorfer and J. Wind, 367-382.

Allen, F. And D. Gale (2000): "Financial contagion," Journal of political economy, 108, $1-33$.

Alvarez, F. And G. Barlevy (2015): "Mandatory Disclosure and Financial Contagion," .

Ambrus, A., W. Y. GaO, And P. Milán (2017): "Informal Risk Sharing with Local Information," .

Ambrus, A., M. Mobius, A. Szeidl, And L. Center (2010): "Consumption risk-sharing in social networks," NBER Working Paper.

Armenter, R. And B. R. Lester (2015): "Excess Reserves and Monetary Policy Normalization," FBR of Philadelphia Working Paper.

Babus, A. (2016): "The formation of financial networks," The RAND Journal of Economics, 47, 239-272.

Barbour, A. D. And P. Hall (1984): "On the rate of Poisson convergence," Mathematical Proceedings of the Cambridge Philosophical Society, 95, 473-480.

Barbour, A. D., L. Holst, And S. Janson (1992): Poisson Approximation, Oxford Studies in Probability 2, Oxford: Clarendon Press.

Battiston, S., D. D. Gatti, M. Gallegati, B. Greenwald, and J. E. Stiglitz (2012): "Liaisons dangereuses: Increasing connectivity, risk sharing, and systemic risk," Journal of Economic Dynamics and Control, 36, 1121-1141.

Bech, M. L. And E. Atalay (2010): "The topology of the federal funds market," Physica A: Statistical Mechanics and its Applications, 389, 5223-5246.

Boss, M., H. Elsinger, M. Summer, And S. T. 4 (2004): "Network topology of the interbank market," Quantitative Finance, 4, 677-684.

Chang, B. And S. Zhang (2016): "Endogenous market making and network formation," .

Chen, L. H. Y. (1975): "Poisson Approximation for Dependent Trials," The Annals of Probability, 3, 534-545. 
Chen, S. X. And J. S. Liu (1997): "Statistical Applications of the Poisson-Binomial and Conditional Bernoulli Distributions," Statistica Sinica, 7, 875-892.

Cocco, J. F., F. J. Gomes, And N. C. Martins (2009): "Lending relationships in the interbank market," Journal of Financial Intermediation, 18, 24 - 48.

Cohen-Cole, E., A. Kirilenko, E. Patacchini, J. Fouque, and J. Langsam (2013): "Strategic interactions on financial networks for the analysis of systemic risk," Handbook on Systemic Risk, 1, 306-326.

Cont, R., A. Moussa, E. Santos, J. Fouque, And J. Langsam (2013): "Handbook on systemic risk," Network structure and systemic risk in banking systems. Cambridge University Press, Cambridge, 327-368.

Craig, B. And G. Von Peter (2014): "Interbank tiering and money center banks," Journal of Financial Intermediation, 23, 322-347.

Duffie, D., N. GÂrleanu, And L. H. Pedersen (2005): "Over-the-Counter Markets," Econometrica, 73, 1815-1847.

Durrett, R. (2007): Random graph dynamics, vol. 200, Cambridge university press Cambridge.

Echenique, F. And A. Wierman (2012): "Finding a Walrasian Equilibrium is Easy for a Fixed Number of Agents," in Proceedings of the 13th ACM Conference on Electronic Commerce, New York, NY, USA: ACM, EC '12, 495-495.

EHм, W. (1991): "Binomial approximation to the Poisson binomial distribution," Statistics 6 Probability Letters, 11, 7 - 16.

EisenberG, L. And T. H. Noe (2001): "Systemic risk in financial systems," Management Science, 47, 236-249.

Elliott, M., B. Golub, And M. O. JaCkson (2014): "Financial Networks and Contagion," American Economic Review, 104, 3115-53.

FARboodi, M. (2015): "Intermediation and Voluntary Exposure to Counterparty Risk," .

Fernández, M. AND S. Williams (2010): "Closed-form expression for the poisson-binomial probability density function," IEEE Transactions on Aerospace and Electronic Systems, 46, 803-817.

Franklin, A., A. Babus, And E. Carletti (2009): "Financial Crises: Theory and Evidence," Annual Review of Financial Economics, 1, 97-116.

Freeman, S. (1996): "The payments system, liquidity, and rediscounting," The American Economic Review, 1126-1138.

Freixas, X., B. M. Parigi, And J.-C. Rochet (2000): "Systemic risk, interbank relations, and liquidity provision by the central bank," Journal of money, credit and banking, 611-638.

Gai, P. And S. Kapadia (2010): "Contagion in financial networks," in Proceedings of the Royal Society of London A: Mathematical, Physical and Engineering Sciences, The Royal Society, vol. 466, 2401-2423.

Gale, D. M. And S. KARIV (2007): "Financial networks," The American economic review, 97, 99-103. 
Green, E. J. AND R. Zhou (2002): "Dynamic monetary equilibrium in a random matching economy," Econometrica, 70, 929-969.

HARSANYi, J. C. (1967): "Games with incomplete information played by ?Bayesian? players, I-III Part I. The basic model," Management science, 14, 159-182.

Hendershott, T. And A. Madhavan (2015): "Click or Call? Auction versus Search in the Over-the-Counter Market," The Journal of Finance, 70, 419-447.

Hojman, D. A. AND A. Szeidl (2008): "Core and periphery in networks," Journal of Economic Theory, 139, 295-309.

Hong, Y. (2013): "On computing the distribution function for the Poisson binomial distribution," Computational Statistics 85 Data Analysis, 59, 41 - 51.

Jackson, M. O. (2008): Social and Economic Networks, Princeton University Press.

JACKSON, M. O. AND A. Wolinsky (1996): "A strategic model of social and economic networks," Journal of economic theory, 71, 44-74.

JARAMILlO, M. (2012): "The spatial geography of teacher labor markets: Evidence from a developing country," Economics of Education Review, 31, 984-995.

Kiyotaki, N. And R. Wright (1989): "On money as a medium of exchange," Journal of Political economy, 97, 927-954.

Langfield, S., Z. LiU, And T. Ota (2014): "Mapping the UK interbank system," Journal of Banking \& Finance, 45, 288-303.

LE CAM, L. (1960): "An approximation theorem for the Poisson binomial distribution." Pacific J. Math., 10, 1181-1197.

Levy, H. And H. Markovitz (1979): "Approximating Expected Utility by a Function of Mean and Variance," The American Economic Review, 69, 308-317.

LI, D. AND N. Schürhoff (2014): "Dealer networks," .

LoIsTL, O. (1976): "The erroneous approximation of expected utility by means of a Taylor's series expansion: analytic and computational results," The American Economic Review, 66, 904-910.

Mas-Colell, A., M. D. Whinston, J. R. Green, et Al. (1995): Microeconomic theory, vol. 1, Oxford university press New York.

Negishi, T. (1960): "Welfare economics and existence of an equilibrium for a competitive economy," Metroeconomica, 12, 92-97.

Samphantharak, K. And R. M. TOWnsend (2010): Households as corporate firms: an analysis of household finance using integrated household surveys and corporate financial accounting, 46, Cambridge University Press.

Santos, M. S. And M. Woodford (1997): "Rational asset pricing bubbles," Econometrica: Journal of the Econometric Society, 19-57.

SAson, I. (2013): "Improved lower bounds on the total variation distance for the Poisson approximation," Statistics \& Probability Letters, 83, 2422 - 2431.

Stein, C. (1986): "Approximate Computation of Expectations," Lecture Notes-Monograph Series, 7, i-164. 
Summer, M. (2013): "Financial Contagion and Network Analysis," Annual Review of Financial Economics, 5, 1-38.

Tishby, I., O. Biham, And E. KAtzaV (2017): "The distribution of first hitting times of randomwalks on Erdős-Rényi networks," Journal of Physics A: Mathematical and Theoretical, $50,115001$.

Trejos, A. And R. Wright (1995): "Search, bargaining, money, and prices," Journal of political Economy, 103, 118-141.

Upper, C. And A. Worms (2004): "Estimating bilateral exposures in the German interbank market: Is there a danger of contagion?" European Economic Review, 48, 827-849.

Volkova, A. Y. (1996): "A Refinement of the Central Limit Theorem for Sums of Independent Random Indicators," Theory of Probability $\&$ Its Applications, 40, 791-794.

\section{Appendix A. Proofs}

Proof of Proposition 3.1. Since $V(t)$ is concave, this program is convex, and satisfies Slater's condition if $T>0$, and hence the Kuhn-Tucker conditions of this program are both necessary and sufficient. The Lagrangian of program 3.1 is $\mathcal{L}(t, \eta, \nu)=V(t)+\eta\left(T-\sum_{j \in J} t_{j}\right)+\nu_{j} t_{j}$. Kuhn-tucker conditions are

(1) $V_{j}(t)+\nu_{j}=\eta$ for all $j \in J$, where $V_{j}=\partial V / \partial t_{j}$

(2) $\nu_{j} t_{j}=0$ for all $j \in J$

(3) $\nu_{j} \geq 0$ for all $j \in J$

(4) $\eta\left(T-\sum_{j \in J} t_{j}\right)=0$ and $\eta \geq 0$

Propose the following solution: $t_{i}^{*}=T, t_{j}^{*}=0$ for all $j \neq i, \eta=V_{i}\left(t^{*}\right)$ and $\nu_{j}=\eta-V_{j}\left(t^{*}\right)$. Since $V$ is differentiable, its partial derivatives are continuous around $t=0$. Therefore, $\exists \hat{T}_{J}>0$ such that for all $t \in \tau=\left\{t: \sum_{j \in J} t_{j}<\hat{T}_{J}\right.$ and $t_{j} \geq 0$ for all $\left.j \in J\right\}$ we have $V_{i}(t)>V_{j}(t)$ for all $j \in J \sim\{i\}$ (since $F C_{i}>F C_{j}$ ). Therefore, if $T<\hat{T}$, a solution $t^{*} \in \tau$, and therefore we have $V_{i}\left(t^{*}\right)>V_{j}\left(t^{*}\right)$ for all $j$, and hence $\nu_{j}=\eta-V_{j}\left(t^{*}\right)=V_{i}\left(t^{*}\right)-V_{j}\left(t^{*}\right)>0$; i.e. $t^{*}$ satisfies the Kuhn-Tucker conditions. To prove uniqueness, suppose there exists another solution $\hat{t}$ : $\sum_{j \in J} \hat{t}_{j}<\hat{T}$ and $\exists k \neq i$ with $\hat{t}_{k}>0$. If that was the case, then $\eta=V_{k}(t)$. But because $\sum_{j \in J} \hat{t}_{j}<\hat{T}$ we also have that $V_{i}(\hat{t})>V_{k}(\hat{t})$. Therefore, $V_{i}(\hat{t})+\nu_{i} \geq V_{i}(\hat{t})>V_{j}(\hat{t})=\eta$, violating condition (1). Therefore, the only solution to 3.1 is $t=t^{*}$

Proof of Proposition 3.2. Using the envelope theorem, and letting $q(s)$ be the Lagrange multipliers of the original program, we get the result.

Proof of Proposition 3.3. We will focus on allocations where $c_{i}(s)>0$ for all $s=(y, \zeta)$ : $\zeta_{i}=1$ for simplicity. Since 3.5 is a convex optimization problem and $u(\cdot)$ is strictly concave and differentiable, Kuhn Tucker conditions are necessary and sufficient to characterize the optimum. This is also true for the planner's problem 2.1. Let $\mu_{i}>0$ be the lagrange multiplier of the AD 
budget constraint in 3.6 (this constraint will always be binding). The first order conditions of the consumer problem with respect to $a_{i}(s)$ at states $s=(y, \zeta): \zeta_{i}=1$

$$
u_{i}^{\prime}\left[c_{i}(s)\right] \mathrm{P}(s)=\mu_{i} r(s) \text { for all } s: \zeta_{i}=1 \text { where } c_{i}(s)=y_{i}(s)+a_{i}(s)
$$

where $c_{i}(s)=y_{i}(s)+a_{i}(s)$. Also see that the choice of $a_{i}(s)$ is superfluous in the consumer's problem if $c_{i}(s)=0$ for all $s: \zeta_{i}=0$, and that the budget constraint can be written as $\sum \zeta_{i} c_{i}(s) r(s) \leq \sum \zeta_{i} y_{i} r(s)$. Hence $c=\left(c_{i}(s)\right)_{i \in I, s \in S}$ is a Walrasian equilibrium with transfers allocation if $\exists \mu_{i}>0 \forall i \in I$ such that conditions A.1 and the resource constraint 2.2 are satisfied, and such that $c_{i}(s)=y_{i}$ for all $s: \zeta_{i}=0$. The corresponding Walrasian Equilibrium has $a_{i}(s)=c_{i}(s)-y_{i}, r(s)=\left(1 / \mu_{i}\right) u_{i}^{\prime}\left[c_{i}(s)\right] \mathrm{P}(s)>0$ and $\tau_{i}=\sum_{s} a_{i}(s) r(s)=$ $\left(1 / \mu_{i}\right) \mathbb{E}_{s}\left\{\left[c_{i}(s)-y_{i}\right] u_{i}^{\prime}\left[c_{i}(s)\right]\right\}$.

Doing the same exercise for the planner's problem 2.1, we get that a consumption allocation $c_{i}(s)$ solves the planner's problem with Pareto Weights $\lambda \in \Delta$ if and only if it satisfies the resource constraint 2.2 for all $s \in S, c_{i}(s)=y_{i}$ for all $s: \zeta_{i}=0$ and all $i \in I$, and satisfies for all $i \in I$ :

$$
\lambda_{i} u_{i}^{\prime}\left[c_{i}(s)\right]=q(s) \text { for all } s: \zeta_{i}=1
$$

where $q(s)$ is the (normalized) lagrange multiplier of the resource constraint at state $s$.

Therefore, a Walrasian Equilibrium with transfers consumption allocation $c$ will also be the solution to the Planner's Problem 2.1 with Pareto Weights $\lambda_{i}=1 / \mu_{i}$. Likewise, for given $\lambda \in \Delta$, the solution to the Planner's Problem 2.1 will be a Walrasian Equilibrium with transfers if we take $\mu_{i}=1 / \lambda_{i}$. Moreover, the implementing price function $r(s)$ and transfers $\tau_{i}$ satisfy:

$$
\begin{gathered}
r(s)=\left(1 / \mu_{i}\right) u_{i}^{\prime}\left[c_{i}(s)\right] \mathrm{P}(s)=q(s) \mathrm{P}(s) \\
\tau_{i}=\mathbb{E}_{s}\left\{\left[c_{i}(s)-y_{i}\right] q(s)\right\}
\end{gathered}
$$

since $1 / \mu_{i}=\lambda_{i}$.

Proof of Subsection 5.1. We use the same approximation around $y=\mu$ of Proposition 4.1 , but now we have that $\mathbb{E}\left\{(\bar{y}-\mu)^{2} \mid \zeta\right\}=\frac{1}{n_{\zeta}^{2}} \sum_{i: \zeta_{i}=1} \sum_{j: \zeta_{j}=1} \sigma_{i j}=\frac{1}{n_{\zeta}} \bar{\sigma}_{\zeta}^{2}$ as defined above. Following the same steps of the proof of Proposition 4.1 we get to equation 5.1

Proof of Subsection 5.2. Take the case of CES preferences: $u(c)=\left(c^{1-\rho}-1\right) /(1-\rho)$. The first order conditions of planner program 2.1 are now

$$
\lambda_{i} c_{i}^{-\rho}=q(s) \Longleftrightarrow c_{i}(s)=\lambda_{i}^{1 / \rho} q(s)^{-1 / \rho}
$$

Putting this equation in the resource constraint of state $s$, and solving for $q(s)$ we get

$$
q(s)^{-1 / \rho} \sum \lambda_{i}^{1 / \rho}=n_{\zeta} \bar{y} \Longleftrightarrow q(s)=\left(\frac{1}{n_{\zeta}} \sum_{j: \zeta_{j}=1} \lambda_{j}^{1 / \rho}\right)^{\rho} \bar{y}^{-\rho}
$$


so $q(s)=\bar{\lambda}_{\zeta} \times u^{\prime}\left(\bar{y}_{\zeta}\right)$, where $\bar{\lambda}_{\zeta}$ is the LP norm of $\lambda_{\zeta}$. We then use the approximation of subsection 5.1 to get the desired result.

For CARA preferences, the first order conditions are

$$
\lambda_{i} \exp \left(-r c_{i}\right)=q(s) \Longleftrightarrow r c_{i}=\ln \left(\lambda_{i}\right)-\ln [q(s)]
$$

Again, using this equation in the resource constraint of state $s$, we solve for $q(s)$ in the same manner as before:

$$
\begin{aligned}
\frac{1}{r} \sum_{j: \zeta_{j}=1} \ln \left(\lambda_{j}\right)-n_{\zeta} \frac{1}{r} \ln [q(s)]=n_{\zeta} \bar{y}_{\zeta} \Longleftrightarrow q(s)=\exp \left[\frac{1}{n_{\zeta}} \sum_{j: \zeta_{j}=1} \ln \left(\lambda_{j}\right)-r \bar{y}_{\zeta}\right] \\
=\underbrace{\exp \left(\frac{1}{n_{\zeta}} \sum_{j: \zeta_{j}=1} \ln \left(\lambda_{j}\right)\right)}_{:=\bar{\lambda}_{\zeta}} \times \underbrace{\exp \left(-r \bar{y}_{\zeta}\right)}_{:=u^{\prime}\left(\bar{y}_{\zeta}\right)}
\end{aligned}
$$

so $q(s)=\bar{\lambda}_{\zeta} u^{\prime}\left(\bar{y}_{\zeta}\right)$ where now $\bar{\lambda}_{\zeta}$ is the geometric mean of Pareto weights in the market. From here on, we follow the proof of the result of subsection 5.2.

Proof of Subsections 5.3 and 5.4. We follow the previous proof above, now with heterogeneous preferences. The first order condition now implies that $r_{i} c_{i}=\ln \left(\lambda_{i}\right)-\ln [q(s)]$. Again substituting this equation into the resource constraint in state $s$, we obtain

$$
\sum_{j: \zeta_{j}=1} r_{j}^{-1} \ln \left(\lambda_{j}\right)-\sum_{j: \zeta_{j}=1} r_{j}^{-1} \ln [q(s)]=n_{\zeta} \bar{y}
$$

and using the definitions $\bar{r}_{\zeta}=\left(\frac{1}{n_{\zeta}} \sum \zeta_{j} \frac{1}{r_{j}}\right)^{-1}$ and $\hat{\lambda}_{\zeta}:=\exp \left(\frac{1}{n_{\zeta}} \sum \zeta_{j} \frac{\bar{r}_{\zeta}}{r_{j}} \ln \left(\lambda_{j}\right)\right)$, we can rewrite this as

$$
\begin{gathered}
\frac{1}{n_{\zeta}} \sum_{j: \zeta_{j}=1} \frac{\bar{r}_{\zeta}}{r_{j}} \ln \left(\lambda_{j}\right)-\ln [q(s)]=\bar{r}_{\zeta} \bar{y} \Longleftrightarrow \\
q(s)=\hat{\lambda}_{\zeta} \exp \left(-\bar{r}_{\zeta} \bar{y}_{\zeta}\right)
\end{gathered}
$$

Since $\zeta \perp y$ we have that $\exp \left(-\bar{r}_{\zeta} \bar{y}_{\zeta}\right) \mid \zeta$ is independent of $\hat{\lambda}_{\zeta} \mid \zeta$. Moreover, because $y \sim$ $\mathcal{N}(\mu, \Sigma)$ we know that $\bar{y} \mid \zeta \sim \mathcal{N}\left(\bar{\mu}_{\zeta}, \frac{1}{n_{\zeta}} \bar{\sigma}_{\zeta}^{2}\right)$, where $\bar{\mu}_{\zeta}:=\frac{1}{n_{\zeta}} \sum \zeta_{j} \mu_{j}$ and $\bar{\sigma}_{\zeta}^{2}:=\frac{1}{n_{\zeta}} \sum \zeta_{j} \zeta_{k} \sigma_{j k}$ as we defined before. Using the moment generating function of the Normal Distribution, we obtain

$$
\mathbb{E}_{y}\left[\exp \left(-\bar{r}_{\zeta} \bar{y}_{\zeta}\right) \mid \zeta\right]=\exp \left(-\bar{r}_{\zeta} \bar{\mu}_{\zeta}\right) \exp \left(\frac{\bar{r}_{\zeta}^{2}}{2} \frac{\bar{\sigma}_{\zeta}^{2}}{n_{\zeta}}\right)
$$

Using equation A.5 in the definition of financial centrality, we obtain:

$$
F C_{i}=\mathbb{E}_{\zeta}\left\{\zeta_{i} \mathbb{E}_{y}[q(y, \zeta) \mid \zeta]\right\}=\mathbb{E}_{\zeta}\left\{\zeta_{i} \mathbb{E}_{y}\left[\hat{\lambda}_{\zeta} \exp \left(-\bar{r}_{\zeta} \bar{y}_{\zeta}\right) \mid \zeta\right]\right\}=
$$




$$
=\mathbb{E}_{\zeta}\left\{\zeta_{i} \hat{\lambda}_{\zeta} \exp \left(-\bar{r}_{\zeta} \bar{\mu}_{\zeta}\right) \exp \left(\frac{\bar{r}_{\zeta}^{2}}{2} \frac{\bar{\sigma}_{\zeta}^{2}}{n_{\zeta}}\right)\right\}
$$

proving the desired result of subsection 5.4. Subsection 5.3 is the special case where $r_{i}=r$, which implies that $\bar{r}_{\zeta}=r$ and $\hat{\lambda}_{\zeta}=\bar{\lambda}_{\zeta}$, the simple geometric average of Pareto weights.

Proof of Proposition 8.1. In the first order conditions of the planner's problem we have

$$
\lambda_{i} \exp \left(-r_{i} c_{i}\right)=\bar{\lambda}_{\zeta} \exp \left(-\bar{r}_{\zeta} \bar{y}\right)
$$

so

Hence

$$
-\frac{1}{r_{i}} \exp \left(-r_{i} c_{i}\right)=-\frac{\bar{\lambda}_{\zeta}}{r_{i} \lambda_{i}} \exp \left(-\bar{r}_{\zeta} \bar{y}\right)
$$

$$
\begin{aligned}
\mathbb{E}_{s}\left[\zeta_{i} u_{i}\left(c_{i}(s)\right)\right] & =-\frac{1}{r_{i} \lambda_{i}} \mathbb{E}_{s}\left[\zeta_{i} \bar{\lambda}_{\zeta} \exp \left(-\bar{r}_{\zeta} \bar{y}\right)\right] \\
& =-\frac{1}{r_{i} \lambda_{i}} \mathbb{E}_{\zeta}\left[\zeta_{i} \bar{\lambda}_{\zeta} \exp \left(-\bar{r}_{\zeta} \bar{\mu}+\frac{\bar{r}_{\zeta}^{2}}{2 n_{\zeta}} \bar{\sigma}_{\zeta}^{2}\right)\right] \\
& =-\frac{1}{r_{i} \lambda_{i}} F C_{i} .
\end{aligned}
$$

Moreover

$$
\begin{aligned}
\mathbb{E}_{s}\left[\zeta_{i} u_{i}\left(y_{i}\right)\right] & =-\frac{1}{r_{i}} \mathbb{E}_{s}\left[\exp \left(-r_{i} y_{i}\right)\right] \\
& =-\frac{1}{r_{i}} \mathbb{E}_{\zeta}\left[\zeta_{i} \exp \left(-r_{i} \mu_{i}+\frac{r_{i}^{2}}{2} \sigma_{i}^{2}\right)\right] \\
& =-\frac{1}{r_{i}} \underbrace{\mathrm{P}\left(\zeta_{i}=1\right)}_{:=p_{i}} \exp \left(-r_{i} \mu_{i}+\frac{r_{i}^{2}}{2} \sigma_{i}^{2}\right) .
\end{aligned}
$$

This means

$$
\mathbb{E}_{s}\left\{\zeta_{i}\left[u_{i}\left(c_{i}(s)\right)-u_{i}\left(y_{i}\right)\right]\right\}=\frac{1}{r_{i}} p_{i} \exp \left(-r \mu_{i}+\frac{r_{i}^{2}}{2} \sigma^{2}\right)-\frac{1}{r_{i} \lambda_{i}} F C_{i}
$$

and so

$$
\begin{gathered}
\lambda_{i}=\frac{\alpha_{i} r_{i}}{\frac{\lambda_{i}}{\lambda_{i}} p_{i} \exp \left(-r_{i} \mu_{i}+\frac{r_{i}^{2}}{2} \sigma_{i}^{2}\right)-\frac{1}{\lambda_{i}} F C_{i}} \Longleftrightarrow \\
\lambda_{i}=\frac{\lambda_{i} \alpha_{i} r_{i}}{\lambda_{i} p_{i} \exp \left(-r_{i} \mu_{i}+\frac{r_{i}^{2}}{2} \sigma_{i}^{2}\right)-F C_{i}} \Longleftrightarrow \lambda_{i} p_{i} \exp \left(-r_{i} \mu_{i}+\frac{r_{i}^{2}}{2} \sigma_{i}^{2}\right)-F C_{i}=\alpha_{i} r_{i} \Longleftrightarrow \\
\lambda_{i}=\frac{\alpha_{i} r_{i}+F C_{i}(\lambda)}{p_{i} \exp \left(-r_{i} \mu_{i}+\frac{r_{i}^{2}}{2} \sigma_{i}^{2}\right)}
\end{gathered}
$$


as we wanted to show. In the first order conditions of the planner's problem we have

$$
\lambda_{i} \exp \left(-r_{i} c_{i}\right)=\bar{\lambda}_{\zeta} \exp \left(-\bar{r}_{\zeta} \bar{y}\right)
$$

so

Hence

$$
-\frac{1}{r_{i}} \exp \left(-r_{i} c_{i}\right)=-\frac{\bar{\lambda}_{\zeta}}{r_{i} \lambda_{i}} \exp \left(-\bar{r}_{\zeta} \bar{y}\right)
$$

$$
\begin{aligned}
\mathbb{E}_{s}\left[\zeta_{i} u_{i}\left(c_{i}(s)\right)\right] & =-\frac{1}{r_{i} \lambda_{i}} \mathbb{E}_{s}\left[\zeta_{i} \bar{\lambda}_{\zeta} \exp \left(-\bar{r}_{\zeta} \bar{y}\right)\right] \\
& =-\frac{1}{r_{i} \lambda_{i}} \mathbb{E}_{\zeta}\left[\zeta_{i} \bar{\lambda}_{\zeta} \exp \left(-\bar{r}_{\zeta} \bar{\mu}+\frac{\bar{r}_{\zeta}^{2}}{2 n_{\zeta}} \bar{\sigma}_{\zeta}^{2}\right)\right] \\
& =-\frac{1}{r_{i} \lambda_{i}} F C_{i} .
\end{aligned}
$$

Moreover

$$
\begin{aligned}
\mathbb{E}_{s}\left[\zeta_{i} u_{i}\left(y_{i}\right)\right] & =-\frac{1}{r_{i}} \mathbb{E}_{s}\left[\exp \left(-r_{i} y_{i}\right)\right]=-\frac{1}{r_{i}} \mathbb{E}_{\zeta}\left[\zeta_{i} \exp \left(-r_{i} \mu_{i}+\frac{r_{i}^{2}}{2} \sigma_{i}^{2}\right)\right] \\
& -\frac{1}{r_{i}} \underbrace{\mathrm{P}\left(\zeta_{i}=1\right)}_{:=p_{i}} \exp \left(-r_{i} \mu_{i}+\frac{r_{i}^{2}}{2} \sigma_{i}^{2}\right) .
\end{aligned}
$$

Therefore

$$
\mathbb{E}_{s}\left\{\zeta_{i}\left[u_{i}\left(c_{i}(s)\right)-u_{i}\left(y_{i}\right)\right]\right\}=\frac{1}{r_{i}} p_{i} \exp \left(-r \mu_{i}+\frac{r_{i}^{2}}{2} \sigma^{2}\right)-\frac{1}{r_{i} \lambda_{i}} F C_{i}
$$

and so

$$
\begin{gathered}
\lambda_{i}=\frac{\alpha_{i} r_{i}}{\frac{\lambda_{i}}{\lambda_{i}} p_{i} \exp \left(-r_{i} \mu_{i}+\frac{r_{i}^{2}}{2} \sigma_{i}^{2}\right)-\frac{1}{\lambda_{i}} F C_{i}} \Longleftrightarrow \\
\lambda_{i}=\frac{\lambda_{i} \alpha_{i} r_{i}}{\lambda_{i} p_{i} \exp \left(-r_{i} \mu_{i}+\frac{r_{i}^{2}}{2} \sigma_{i}^{2}\right)-F C_{i}} \Longleftrightarrow \lambda_{i} p_{i} \exp \left(-r_{i} \mu_{i}+\frac{r_{i}^{2}}{2} \sigma_{i}^{2}\right)-F C_{i}=\alpha_{i} r_{i} \Longleftrightarrow \\
\lambda_{i}=\frac{\alpha_{i} r_{i}+F C_{i}(\lambda)}{p_{i} \exp \left(-r_{i} \mu_{i}+\frac{r_{i}^{2}}{2} \sigma_{i}^{2}\right)}
\end{gathered}
$$

as we wanted to show.

Proof of Proposition 8.2. The Lagrangian is

$\mathcal{L}=\alpha+\sum \mu_{i}\left\{\mathbb{E}_{y, \zeta}\left[\zeta_{i} u_{i}\left(c_{i}\right)+\left(1-\zeta_{i}\right) u_{i}\left(y_{i}\right)\right]-\alpha \bar{U}_{i}-(1-\alpha) \underline{U}_{i}\right\}+\sum_{\zeta, y} q(y, \zeta) \zeta_{i}\left(y_{i}-c_{i}\right) \mathrm{P}(y, \zeta)$ with multipliers $\left(\mu_{i}\right)_{i=1: n}$ and $(q(y, \zeta) \mathrm{P}(y, \zeta))_{y, \zeta}$. First order conditions are

$$
\frac{\partial \mathcal{L}}{\partial \alpha}=1-\mu_{i}\left(\bar{U}_{i}-\underline{U}_{i}\right)
$$


since $\alpha \in(0,1)$ (the bliss point cannot be feasible) then, to get an interior solution, we must have $\frac{\partial \mathcal{L}}{\partial \alpha}=0 \Longleftrightarrow \mu_{i}=1 /\left(\bar{U}_{i}-\underline{U}_{i}\right)$. The first order conditions with respect to consumption are

$$
\left.\frac{\partial \mathcal{L}}{\partial c_{i}(y, \zeta)}\right|_{\zeta_{i}=1}=0 \Longleftrightarrow \mu_{i} u_{i}^{\prime}\left(c_{i}\right) \mathrm{P}(y, \zeta)=q(y, \zeta) \mathrm{P}(y, \zeta)
$$

therefore, in the planer representation, this equivalent to the Pareto weights being

$$
\lambda_{i}=\mu_{i}=\frac{1}{\bar{U}_{i}-\underline{U}_{i}} .
$$

In the CARA Normal model, let $Y:=\sum \zeta_{j} y_{j}$. Since $y \sim \mathcal{N}(\mu, \Sigma)$, we have $Y \mid \zeta \sim$ $\mathcal{N}\left(\sum \zeta_{j} \mu_{j}, \sum_{i, j} \zeta_{i} \zeta_{j} \sigma_{i j}\right)$. Therefore

$$
\mathbb{E}_{y}\left[u_{i}\left(\sum_{i} \zeta_{j} y_{j}\right) \mid \zeta_{i}=1\right]=-\frac{1}{r} \mathbb{E}[\exp (-r Y)]=-\frac{1}{r} M_{Y}(-r)
$$

and $M_{Y}(t)=\exp \left(\mu_{Y} t+\frac{t^{2}}{2} \sigma_{Y}^{2}\right)=\exp \left(-r \mu_{Y}+\frac{r^{2}}{2} \sigma^{2}\right)=\exp \left(-r \times n_{\zeta} \bar{\mu}_{\zeta}+\frac{r^{2}}{2} \sum \sigma_{i j}\right)$. In the i.i.d case, $M_{Y}(t)=\exp \left(-r n_{\zeta} \mu+\frac{r^{2}}{2} \sigma^{2} n_{\zeta}\right)=\exp \left[n_{\zeta}(-r \mu+\gamma)\right]$ and the autarky value is $\mathbb{E}_{y}\left[u\left(y_{i}\right)\right]=\frac{-1}{r} M_{y_{i}}(-r)=\exp \left(-r \mu+\frac{r^{2}}{2} \sigma^{2}\right)$

Therefore

$$
\begin{aligned}
\bar{U}_{i}-\underline{U}_{i} & =\mathbb{E}\left\{\zeta_{i}\left[u_{i}\left(\sum_{j: \zeta_{j}=1} y_{j}\right)-u_{i}\left(y_{i}\right)\right]\right\}=p_{i} \mathbb{E}_{y, \zeta}\left\{u_{i}\left(\sum_{j: \zeta_{j}=1} y_{j}\right)-u_{i}\left(y_{i}\right) \mid \zeta_{i}=1\right\} \\
& =p_{i} \times\left\{\exp \left[n_{\zeta}\left(-r \mu+\frac{r^{2}}{2} \sigma^{2}\right)\right]-\exp \left(-r \mu+\frac{r^{2}}{2} \sigma^{2}\right)\right\}
\end{aligned}
$$

proving the desired result.

Proofs of Subsection 10.2. First, we want to show equation 10.2. For that, we use again the first order conditions of planner's problem 2.1 but with representing pare-to weights $\varphi \geq 0$ : $\varphi_{j} u_{j}^{\prime}\left(c_{j}(s)\right)=q(s) \Longleftrightarrow \lambda_{j} u_{j}^{\prime}\left(c_{j}(s)\right)=\rho_{j} q(s)$ where $\rho_{j}=\lambda_{j} / \varphi_{j}$. Using this in the original definition of centrality in this setup, we get

$$
\begin{aligned}
F C_{i} & =\mathbb{E}_{\zeta}\left\{\zeta_{i} \sum_{j: \zeta_{j}=1} \lambda_{j} u^{\prime}\left(c_{j}(s)\right) \frac{\partial c_{j}(s)}{\partial y_{i}}\right\}=\mathbb{E}_{\zeta}\left\{\zeta_{i} \sum_{j: \zeta_{j}=1} \rho_{j} q(s) \frac{\partial c_{j}(s)}{\partial y_{i}}\right\} \\
& =\mathbb{E}_{\zeta}\left\{\zeta_{i} q(s) \sum_{j: \zeta_{j}=1} \rho_{j} \frac{\partial c_{j}(s)}{\partial y_{i}}\right\},
\end{aligned}
$$

showing the desired result. Also, because the resource constraint is always binding at every state $s$, we have the identity $\sum_{j: \zeta_{j}=1} c_{j}(s)=\sum_{j: \zeta_{j}=1} y_{j}$, which at states $s: \zeta_{i}=1$ implies that $\sum_{j: \zeta_{j}=1} \partial c_{j}(s) / \partial y_{i}=1$. Therefore, if $\lambda=\varphi$, then $\rho_{j}=1 \forall j, q(s)$ is the multiplier for the Pareto problem with Pareto weights $\lambda=\varphi$ and hence, $F C_{i}=\left\{\zeta_{i} q(s)\right\}$, like we had above.

and (2) the resource constraint 
We now study the special case of the CARA+Normal model with homogeneous preferences and a representing Pareto weight vector $\varphi$. We know (see B ) that in this model, $c_{j}(s)=$ $r^{-1} \ln \left(\varphi_{j} / \bar{\varphi}_{\zeta}\right)+\bar{y}$, where $\bar{\varphi}_{\zeta}=\exp \left(n_{\zeta}^{-1} \sum \zeta_{j} \ln \varphi_{j}\right)$. This then means that whenever $\zeta_{i}=$ $\zeta_{j}=1$, we have $\partial c_{j}(s) / \partial y_{i}=n_{\zeta}^{-1}$. Moreover, we also showed that in this environment, $q(s)=\bar{\varphi}_{\zeta} \exp (-r \bar{y})$. Therefore, using 10.2 we get $F C_{i}=\mathbb{E}_{s}\left\{\zeta_{i} \bar{\varphi}_{\zeta} \exp (-r \bar{y}) \times \bar{\rho}_{\zeta}\right\}$, where now $\bar{\rho}_{\zeta}:=n_{\zeta}^{-1} \sum \zeta_{j} \rho_{j}$ is the arithmetic mean of relative Pareto weights. Using the assumption $y \perp \zeta$, we can then rewrite it as

$$
\mathbb{E}_{s}\left\{\zeta_{i} \bar{\varphi}_{\zeta} \exp \left(-r \bar{\mu}_{\zeta}\right) \exp \left(\gamma \frac{\bar{\sigma}_{\zeta}^{2}}{n_{\zeta}}\right) \times \bar{\rho}_{\zeta}\right\}
$$




\section{Appendix B. Walrasian Equilibrium without Transfers}

Following the definitions in Subsection 3.2, and given a (normalized) price function $r \in$ $\Delta(S)$, we can simplify the consumer's problem by just choosing consumption to maximize utility, given only one "expected" budget constraint. Formally, agent $i \in\{1, \ldots, n\}$ solves

$$
\begin{gathered}
V_{i}(q):=\max \mathbb{E}_{s}\left\{\zeta_{i} u_{i}\left[c_{i}(s)\right]+\left(1-\zeta_{i}\right) u_{i}\left(y_{i}\right)\right\} \\
\text { subject to: } \mathbb{E}_{s}\left[\zeta_{i} c_{i}(s) r(s)\right] \leq \mathbb{E}_{s}\left[\zeta_{i} y_{i} r(s)\right]
\end{gathered}
$$

As we did when defining the Lagrange multipliers for the planning problem, we normalize the price function as $q(s) \mathrm{P}(s)=\hat{q}(s)$, where $\hat{q}$ is the actual price measure. A Walrasian equilibrium is a pair $(c, q)=\left(\left\{c_{i}(s)\right\}_{i \in I, s \in S},\{q(s)\}_{s \in S}\right)$ such that

- $\left\{c_{i}(s)\right\}_{s \in S}$ solves B.1 given prices $q(s)$

- and markets clear at all states: $\sum_{i} \zeta_{i} c_{i}(s) \leq \sum_{i} \zeta_{i} y_{i}$. for all $s=(y, \zeta)$

Proposition ?? implies there exist a vector $\lambda$ such that the equilibrium allocation solves the planning problem 2.1, and such that the normalized prices satisfy $r(s)=q(s)$, where $q(s)$ are the normalized lagrange multipliers of the resource constraint at state $s$. Following Negishi (1960) and more recently Echenique and Wierman (2012), we can then solve for the equilibrium allocation by finding the Pareto weights that satisfy the budget constraints for all agents. Formally, let $c_{i}^{*}(s \mid \lambda)$ be the optimal consumption allocation in the planning problem with weights $\lambda$, and $q^{*}(s \mid \lambda)$ the Lagrange multipliers (normalized by the probabilities of each state). Then, a Pareto weight vector $\lambda$ corresponds to a Walrasian equilibrium allocation if and only if

$$
\mathbb{E}_{s}\left[\zeta_{i} c_{i}^{*}(s \mid \lambda) q^{*}(s \mid \lambda)\right]=\mathbb{E}_{\zeta}\left[\zeta_{i} y_{i} q^{*}(s \mid \lambda)\right] \text { for all } i=1,2 \ldots n
$$

The next proposition characterizes the Pareto weights equation for the CARA normal case

Proposition B.1. Suppose $u_{i}(c)=-r_{i}^{-1} \exp \left(-r_{i} c\right)$ and $y \sim \mathcal{N}(\mu, \Sigma)$. Let $\bar{r}_{\zeta}:=\left(\frac{1}{n_{\zeta}} \sum \zeta_{i} r_{i}^{-1}\right)^{-1}$ be the harmonic mean of risk aversion in market $\zeta$, and $\bar{\lambda}_{\zeta}:=\exp \left[\frac{1}{n_{\zeta}} \sum_{i} \zeta_{i}\left(\bar{r}_{\zeta} / r_{i}\right) \ln \left(\lambda_{i}\right)\right]$ be the average Pareto weight in the market, weighted by the relative risk aversion. Also, let $\Sigma_{i, \zeta}:=\sum_{j} \zeta_{j} \sigma_{i j}$ Then the Pareto weight vector $\lambda$ solving B.2 satisfies:

$$
\ln \left(\lambda_{i}\right)=\frac{\mathbb{E}_{\zeta}\left\{\zeta_{i}\left[\ln \left(\bar{\lambda}_{\zeta}\right)+\left(r_{i} \mu_{i}-\bar{r}_{\zeta} \bar{\mu}_{\zeta}\right)-\frac{\bar{r}_{\zeta}}{n_{\zeta}}\left(r_{i} \Sigma_{i, \zeta}-\bar{r}_{\zeta} \bar{\sigma}_{\zeta}^{2}\right)\right] \eta(s)\right\}}{\mathbb{E}_{\zeta}\left\{\zeta_{i} \eta(s)\right\}}
$$

for $i=1, \ldots n$, where $\eta(s):=\bar{\lambda}_{\zeta} \exp \left(-\bar{r}_{\zeta} \bar{\mu}_{\zeta}+\frac{\bar{r}_{\zeta}^{2}}{2} \frac{\bar{\sigma}_{\zeta}^{2}}{n_{\zeta}}\right)$.

Proof. From the first order conditions under CARA preferences, we get

$$
\lambda_{i} \exp \left(-r_{i} c_{i}\right)=q(s) \Longleftrightarrow c_{i}=\frac{1}{r_{i}} \ln \left(\lambda_{i}\right)-\frac{1}{r_{i}} \ln (q(s))
$$

and that

$$
q(s)=\bar{\lambda}_{\zeta} \exp \left(-\bar{r}_{\zeta} \bar{y}\right)=\bar{\lambda}_{\zeta} \exp \left(-\bar{r}_{\zeta} \bar{y}\right)
$$


Using the first order conditions again, whenever $\zeta_{i}=1$ we get

$$
c_{i}(s)=\frac{\ln \left(\lambda_{i} / \bar{\lambda}_{\zeta}\right)}{r_{i}}+\frac{\bar{r}_{\zeta}}{r_{i}} \bar{y}(s)
$$

Then, the value of the consumption allocation, at prices $q(s)$ is

$$
\begin{gathered}
\mathbb{E}_{s}\left\{\zeta_{i} c(s) q(s)\right\}=\mathbb{E}_{s}\left[\zeta_{i} \frac{\ln \left(\lambda_{i}\right)}{r_{i}} q(s)\right]-\mathbb{E}_{s}\left\{\zeta_{i} \frac{\ln \left(\bar{\lambda}_{\zeta}\right)}{r_{i}} q(s)\right\}+\mathbb{E}_{s}\left\{\zeta_{i} \frac{\bar{r}_{\zeta}}{r_{i}} \bar{y}(s) q(s)\right\}= \\
\frac{1}{r_{i}} \ln \left(\lambda_{i}\right) F C_{i}(\lambda)-\frac{1}{r_{i}} \mathbb{E}_{s}\left\{\zeta_{i} \bar{\lambda}_{\zeta}\left[\ln \left(\bar{\lambda}_{\zeta}\right)+\bar{r}_{\zeta} \bar{y}\right] \exp \left(-\bar{r}_{\zeta} \bar{y}\right)\right\}
\end{gathered}
$$

where $\mathbb{E}_{y}\left(-\bar{r}_{\zeta} \bar{y}\right)=\exp \left(-\bar{r}_{\zeta} \bar{\mu}_{\zeta}+\frac{\bar{r}_{\zeta}}{2} \frac{\bar{\sigma}_{\zeta}}{n_{\zeta}}\right)$ as we have seen before. Moreover

$$
\mathbb{E}_{s}\left[\bar{r}_{\zeta} \bar{y} \exp \left(-\bar{r}_{\zeta} \bar{y}\right)\right]=\mathbb{E}_{\zeta}\left[\left(\bar{r}_{\zeta} \bar{\mu}_{\zeta}-\frac{\bar{r}_{\zeta}^{2}}{n_{\zeta}} \bar{\sigma}_{\zeta}^{2}\right) \exp \left(-\bar{r}_{\zeta} \bar{\mu}_{\zeta}+\frac{r_{\zeta}^{2}}{2} \frac{\bar{\sigma}^{2}}{n_{\zeta}}\right)\right]
$$

On the other hand, the value of agent $i$ ' $\mathrm{s}$ income stream is

$$
w_{i}=\mathbb{E}_{\zeta}\left[\zeta_{i} y_{i} q(s)\right]=\mathbb{E}_{s}\left\{\zeta_{i} \bar{\lambda}_{\zeta} y_{i} \exp \left[-\bar{r}_{\zeta} \bar{y}(s)\right]\right\}
$$

Using the moment generating function $M_{y}(t)=\mathbb{E}_{y}\left[\exp \left(t^{\prime} y\right)\right]=\exp \left(t^{\prime} \mu+\frac{1}{2} t^{\prime} \Sigma t\right)$, we get $\mathbb{E}\left[y_{i} \exp \left(-\bar{r}_{\zeta} \bar{y}_{\zeta}\right)\right]=\left.\frac{\partial M}{\partial t_{i}}\right|_{t=-\frac{\bar{y}_{\zeta}}{n_{\zeta}} \times \mathbf{1}}$ where $\mathbf{1}$ is a vector of 1 's, so that $t^{\prime} y=\bar{r}_{\zeta} \bar{y}$. This then implies that $\mathbb{E}_{y}\left[y_{i} \exp \left(-\bar{r}_{\zeta} \bar{y}\right)\right]=\left(\mu_{i}-\frac{\bar{r}_{\zeta}}{n_{\zeta}} \Sigma_{i, \zeta}\right) \exp \left(-\bar{r}_{\zeta} \bar{\mu}_{\zeta}+\frac{\bar{r}_{\zeta}}{2 n_{\zeta}} \bar{\sigma}_{\zeta}^{2}\right)$, where $\Sigma_{i, \zeta}:=\sum_{j} \zeta_{j} \sigma_{i j}$. Putting all this results together, we can write the budget constraint as

$$
\begin{aligned}
& \mathbb{E}_{s}\left[\zeta_{i}\left(c_{i}-y_{i}\right) q(s)\right]=0 \Longleftrightarrow r_{i}^{-1} \ln \left(\lambda_{i}\right) F C_{i}(\lambda)-r_{i}^{-1} \mathbb{E}_{\zeta}\left\{\zeta_{i} \bar{\lambda}_{\zeta}\left[\ln \left(\bar{\lambda}_{\zeta}\right)-\bar{r}_{\zeta} \bar{\mu}_{\zeta}+\frac{\bar{r}_{\zeta}^{2}}{n_{\zeta}} \bar{\sigma}_{\zeta}^{2}\right] \exp \left(-\bar{r}_{\zeta} \bar{\mu}_{\zeta}+\frac{\bar{r}_{\zeta}^{2}}{2 n_{\zeta}} \bar{\sigma}_{\zeta}^{2}\right)\right) \\
& \mathbb{E}_{\zeta}\left\{\zeta_{i} \bar{\lambda}_{\zeta}\left(\mu_{i}-\frac{\bar{r}_{\zeta}}{n_{\zeta}} \Sigma_{i, \zeta}\right) \exp \left(-\bar{r}_{\zeta} \bar{\mu}_{\zeta}+\frac{\bar{r}_{\zeta}^{2}}{2 n_{\zeta}} \bar{\sigma}_{\zeta}^{2}\right)\right\} \Longleftrightarrow \\
& \ln \left(\lambda_{i}\right) F C_{i}(\lambda)=\mathbb{E}_{\zeta}\left\{\zeta_{i} \bar{\lambda}_{\zeta}\left[\ln \left(\bar{\lambda}_{\zeta}\right)+\left(r_{i} \mu_{i}-\bar{r}_{\zeta} \bar{\mu}_{\zeta}\right)-\frac{\bar{r}_{\zeta}}{n_{\zeta}}\left(r_{i} \Sigma_{i, \zeta}-\bar{r}_{\zeta}^{2} \bar{\sigma}_{\zeta}^{2}\right)\right] \exp \left(-\bar{r}_{\zeta} \bar{\mu}_{\zeta}+\frac{\bar{r}_{\zeta}^{2}}{2 n_{\zeta}} \bar{\sigma}_{\zeta}^{2}\right)\right\}
\end{aligned}
$$

See that the denominator $\mathbb{E}_{\zeta}\left\{\zeta_{i} \eta(s)\right\}=F C_{i}$. Also, because $\lambda \in \Delta$, we have $\ln \left(\lambda_{i}\right)$ and $\ln \left(\bar{\lambda}_{\zeta}\right)<0$, which implies that if we could, somehow, increase $F C_{i}$ without affecting the numerator of the right hand side of B.3, would increase $\lambda_{i}$ in the fixed point equation. An important corollary of Proposition B.1 is the proof of Proposition 3.2, since we would have $\bar{r}_{\zeta}=r_{i}=r$ for all $\zeta$, and the fact that incomes are identically distributed and independent imply $\bar{\sigma}_{\zeta}^{2}=\sigma^{2}, \bar{\mu}_{\zeta}=\mu$ and $\Sigma_{i, \zeta}=\sigma^{2}$. This simplifies the fixed point equation as

$$
\ln \left(\lambda_{i}\right) F C_{i}(\lambda)=\mathbb{E}_{\zeta}\left\{\zeta_{i} \bar{\lambda}_{\zeta} \ln \left(\bar{\lambda}_{\zeta}\right) \exp \left(-r \mu+\frac{r^{2}}{2} \frac{\sigma^{2}}{n_{\zeta}}\right)\right\}
$$

to which a solution is $\lambda_{i}=1 / n$. We summarize this result in Proposition B.2 
Proposition B.2. Suppose $u_{i}(c)=-r^{-1} \exp (-r c)$ and $y_{i} \sim_{i . i . d} \mathcal{N}\left(\mu, \sigma^{2}\right)$. Then $\lambda_{i}=1 / n \forall i$ solves B.2, and hence $F C_{i}(\lambda)=\exp (-r \mu) \mathbb{E}_{\zeta}\left\{\zeta_{i} \exp \left(\frac{r^{2}}{2} \frac{\sigma^{2}}{n_{\zeta}}\right)\right\}$. 


\section{Appendix C. Transaction Chains}

A simple transaction chain is a set of agents that can only trade with adjacent agents. Namely, there is a set of agents $J=\left\{i_{1}, i_{2}, \ldots, i_{k}\right\} \in I$ (which are selected randomly), such that $i_{j}$ can trade only with agents $i_{j-1}$ and $i_{j+1}$, for $j \in\{0,1, \ldots, k\}$ (except for the first agent $i_{1}$, who can only trade with $i_{2}$, and the last member $i_{k}$, who can only trade with $i_{k-1}$ ). Suppose

(1) Each agent has an endowment of $y_{j} \geq 0$.

(2) Agent $j$ can make or receive transfers $\hat{T}_{j, h} \in \mathbb{R}$ for $h \in\{j-1, j+1\}$, which might be such that $\hat{T}_{j, h}+y_{j}<0$ (i.e. giving agent $h$ more than the endowment she has at the moment of the transaction). If $\hat{T}_{j, h}>0$ it means that $j$ sends resources to agent $h$, while $\hat{T}_{j, h}<0$ means that $j$ receives resources from $k$. The budget constraint that $j$ faces is then

$$
T_{j, j-1}+T_{j-1, j}+T_{j, j+1}+T_{j+1, j} \leq y_{j}
$$

If we define $T_{j, h}$ as net transfers instead of gross transfers, then $T_{j, j+1}=-T_{j+1, j}$. Therefore, we can work only with the net transfers $T_{j}=T_{j, j+1}$ for agents $j=1,2, \ldots k-$ 1. Therefore the budget constraint is, for every $j=1, \ldots, k-1$

$$
T_{j} \leq y_{j}+T_{j-1}
$$

(3) There is a clearing house that, at the end of the day, settle all transactions. That is, payments are settled simultaneously, once all transactions are agreed upon.

Without loss of generality, let's assume $i_{j}=j$, so that $C=\{1,2, \ldots k\}$. A consumption profile of the agents in the chain $C$, is a description of consumption amounts $c=\left(c_{1}, c_{2}, \ldots c_{k}\right)$. A consumption bundle is feasible if and only if

$$
\sum_{i=1}^{k} c_{i}=\sum_{i=1}^{k} y_{i}
$$

We say that a consumption bundle is transfer-feasible $\Longleftrightarrow$ it is feasible and there exist transfers $\left\{T_{i, j}\right\}_{i=1}^{n}$ such that

$$
c_{j}=y_{j}+T_{j-1}-T_{j} \geq 0
$$

and

$$
\sum_{j=1}^{k-1}\left(T_{j-1}-T_{j}\right)=0
$$

In order to be able to define this objects for all $j$, we set $T_{1-1}=T_{k, k+1}=0$. Therefore, for $i=1$

$$
c_{1}=y_{1}-T_{2}
$$

and for $i=k$

$$
c_{k}=y_{k}+T_{k-1}
$$

For such a consumption allocation, we say the sequence of net transfers $\left\{T_{j}\right\}$ implements the allocation $c$. The (rather obvious) result is that the set of feasible consumption profiles 
is equal to the set of transfer feasible allocations. This then implies that by modeling the interactions among agents as trades "as if" everyone was trading with each other is just an useful representation.

So, the basic assumptions in this environment is that (1) agents can only trade bilaterally with adjacent agents (with a predetermined order) in the chain and (2) promises to pay (i.e. net transfers) have to be settled jointly, after all trades have been agreed upon. This is the most important assumption; which abstracts away from leverage or run-away constraints (which would limit the short-run deficits agents can have in any given moment ). In Proposition C.1 we show that, if we allow agents to run short-run deficits (until the end of the day, where all transactions are settled), than any feasible consumption allocation among $k$ agents can be implemented by a trading chain (in no particular order of agents).

Proposition C.1. Let $c=\left(c_{i}\right)_{i=1}^{i=k}$ be a feasible consumption allocation (so $\sum_{i} c_{i}=\sum_{i} y_{i}$ ). Then, the net transfers $T_{j}$ defined as

$$
T_{j}=T_{j \rightarrow j+1}:=\sum_{i=1}^{i=j}\left(y_{i}-c_{i}\right)
$$

implement c. Moreover, the following gross transfers implement $c$

$$
\hat{T}_{j \rightarrow j+1}=\max \left\{0, T_{j}\right\} \text { and } \hat{T}_{j+1 \rightarrow j}=\max \left\{0,-T_{j}\right\}
$$

so either $\hat{T}_{j \rightarrow j+1}=T_{j}>0$ and $\hat{T}_{j+1 \rightarrow j}=0$, or $\hat{T}_{j \rightarrow j+1}=0$ and $\hat{T}_{j+1 \rightarrow j}=-T_{j} \geq 0$

Proof. The fact that $\sum_{j=1}^{k}\left(T_{j-1}-T_{j}\right)$ comes from equation C.1: we have

$$
T_{j-1}-T_{j}=\sum_{i=1}^{i=j-1}\left(y_{i}-c_{i}\right)-\sum_{i=1}^{i=j}\left(y_{i}-c_{i}\right)=c_{j}-y_{j}
$$

and hence

$$
\sum_{j=1}^{k}\left(T_{j-1}-T_{j}\right)=\sum_{j=1}^{k}\left(c_{j}-y_{j}\right)=0
$$

since $c$ is feasible. The consumption attained for each agent is

$$
\hat{c}_{j}=y_{j}+T_{j-1}-T_{j}=y_{j}+\left(c_{j}-y_{j}\right)=c_{j}
$$

i.e. it achieves the target consumption allocation. 


\section{Appendix D. Endogenous Market Participation: Matching Probability Choice}

We briefly set up another example of endogenous market participation, without fully analyzing it, which concerns moral hazard. Here assume that $y$ is realized and every agent $i$ observes only $z_{i}$, an imperfect signal about $y$ (i.e., $z_{i} \sim \pi_{i}\left(z_{i} \mid y\right)$ for some conditional $\left.\operatorname{cdf} \pi_{i}\right)$. Given this private information, agents simultaneously choose the probability of accessing the market, denoted by $p_{i}\left(z_{i}\right) \in[0,1]=\mathrm{P}\left(\zeta_{i}=1\right)$. Agents have to pay a disutility cost $\psi(p)$, where $\psi$ is strictly increasing and convex.

Given the profile of functions $\left(p_{i}: Z_{i} \rightarrow[0,1]\right)_{i=1}^{n}$, the joint probability of market participation, given income draws, is given by

$$
\mathrm{P}(\zeta \mid z)=\prod_{i=1}^{n}\left[p_{i}\left(z_{i}\right)\right]^{\zeta_{i}}\left[1-p_{i}\left(z_{i}\right)\right]^{1-\zeta_{i}} .
$$

Then consumption is realized according to a feasible consumption allocation $\hat{c}(s)=\zeta_{i} c_{i}(s)+$ $\left(1-\zeta_{i}\right) y_{i}$, where $c_{i}(\cdot)$ is an (eqm) feasible allocation. For this example, we leave unspecified the choice of the consumption allocation, and it is only assumed that the consumption allocation as a function of the state $s=(y, \zeta)$ is common knowledge among agents.

Agents preferences (given $\left.p_{i}(\cdot)\right)$ are

$$
\begin{aligned}
U_{i}\left(y_{i}, p_{i} \mid\left(p_{j}(\cdot)\right)_{j \neq i}\right) & =p_{i} \mathbb{E}_{t_{-i}, s}\left\{\sum_{\zeta_{-i}} \prod_{j \neq i}\left[p_{j}\left(z_{j}\right)\right]^{\zeta_{j}}\left[1-p_{j}\left(z_{j}\right)\right]^{1-\zeta_{j}} u_{i}\left[c_{i}\left(y_{i}, y_{-i}, \zeta_{i}=1, \zeta_{-i}\right)\right] \mid z_{i}\right\} \\
& +\left(1-p_{i}\right) u_{i}\left(y_{i}\right) \\
& -\psi\left(p_{i}\right)
\end{aligned}
$$

As in the private information example in section Again the solution concept is the BNE, with $p^{*}(t)=\left(p_{i}^{*}\left(z_{i}\right)\right)_{i \in N}$ such that for all $i$ and all $y_{i} \in Y$ :

$$
p_{i}^{*}\left(z_{i}\right) \in \underset{p_{i} \in[0,1]}{\operatorname{argmax}} U_{i}\left(y_{i}, p_{i} \mid\left\{p_{j}^{*}(\cdot)\right\}_{j \neq i}\right) .
$$

